\title{
The Relativistic Polaron without Cutoffs
}

\author{
Leonard Gross * \\ Cornell University, Department of Mathematics, Ithaca, N.Y., USA
}

Received July 18, 1972

\begin{abstract}
The (non-Lorentz covariant) system consisting of a relativistic scalar Boson field $\varphi$ interacting with a single spinless particle (relativistic polaron) with kinetic energy function $\left(m^{2}+|p|^{2}\right)^{1 / 2}$ is studied in $d$ space demensions, where $d \geqq 3$. The interaction Hamiltonian is taken to be $\int \Psi(x)^{*} \Psi(x) \varphi(x) d x$ where $\varphi$ has a momentum cutoff. The physical one polaron Hilbert space $\mathscr{K}_{p h}$ for this model, corresponding to no cutoff on $\varphi$, is constructed. The total renormalized Hamiltonian $H$ without cutoff is constructed as a semibounded self-adjoint operator on $\mathscr{K}_{p h}$. The time zero physical Boson field is also constructed. First order estimates are established for the local (in momentum space) number operators in terms of $H$.
\end{abstract}

\section{Introduction}

We consider a single polaron (that is, a spinless electron) interacting with a relativistic scalar Boson field in $d$ space dimensions. We shall be concerned primarily with the cases $d \geqq 3$. We take the kinetic energy function of the polaron to be

$$
E(q)=\left(m^{2}+|q|^{2}\right)^{1 / 2}
$$

where $m$ is the bare mass of the polaron. The total Hamiltonian of the system in the presence of a momentum cutoff $f$ is

$$
\begin{aligned}
& \qquad H_{f}=H_{0}+H_{I}(f) \\
& \begin{aligned}
\text { where } & \\
H_{0} & =\int_{E_{d}} \Psi(p)^{*} E(p) \Psi(p) d p+\int_{E_{d}} a(k)^{*} \mu(k) a(k) d k
\end{aligned} \\
& H_{I}(f)=\int_{E_{d}}\left\{\Psi(p+k)^{*} \Psi(p) a(k)+\Psi(p-k)^{*} \Psi(p) a(k)^{*}\right\} f(k) d k d p \\
& \left.\mu(k)=\left(\mu_{0}^{2}+|k|^{2}\right)^{1 / 2}, \mu_{0}\right\rangle 0
\end{aligned}
$$

and $\Psi(p)$ and $a(k)$ are the annihilation operators for the polaron and Boson field respectively. $E_{d}$ is $d$-dimensional Euclidean space. Our objective is to construct the one polaron physical Hilbert space $\mathscr{K}_{p h}$ associated with this model and to show that the total Hamiltonian

* This research was partially supported by N.S.F. grants GP 28109 and GP 28443 and U.S.A.F. grant AF-AFOSR 743-67. 
without cutoff (i.e. with $f(k)=\lambda \mu(k)^{-1 / 2}$ ) can be defined on this space as a non-trivial semi-bounded self-adjoint operator with lower bound equal to the physical mass $m_{0}$ of the polaron. We also construct the time zero Boson fields as operators on $\mathscr{K}_{p h}$. This model is well suited for studying the phenomenon of infinite field strength renormalization.

We outline the procedure here. Let $f_{n}$ denote a sequence of smooth cutoff functions with compact support. We shall allow $f_{n}$ to converge in a suitable sense to $\lambda \mu(k)^{-1 / 2}$ where $\lambda$ is a real constant. Write $H_{n}$ for the corresponding total Hamiltonian as given by (1.2). We chose the bare polaron mass $m$ so that the lower bound of $H_{n}$ is exactly the physical mass, $m_{0}$ (a given positive constant), of the polaron. As $n \rightarrow \infty$ the bare mass $m$ (which depends on $n$ ) will go to infinity when $d \geqq 2$. Thus the model exhibits infinite mass renormalization when $d \geqq 2$. The bare Hilbert space for the system is

$$
\mathscr{K}=L^{2}\left(E_{d}\right) \otimes \mathscr{F}
$$

where $\mathscr{F}$ is the Fock space for the Boson field and $E_{d}$ is momentum space for the polaron. Since the theory is translation invariant $\mathscr{K}$ decomposes into a direct integral of (infinitesimal) subspaces $\mathscr{F}_{p}$, on which the total momentum of the polaron plus Boson field has the constant value $p$, and which reduce $H_{n}$. Denoting by $H_{n}(p)$ the restriction of $H_{n}$ to $\widetilde{F}_{p}$ we let $\psi_{n}$ be the unique lowest proper vector for $H_{n}(0)$ in $\mathscr{F}_{0}$. The existence and uniqueness of $\psi_{n}$ was shown in [8, Theorem 8]. We take $\psi_{n}$ to be a unit vector. $\psi_{n}$ is the physical rest state of the system with cutoff $f_{n}$ and the corresponding eigenvalue of $H_{n}(0)$ is $m_{0}$.

When $d=2$ one expects, on the basis of perturbation theory, as well as on experience with the external source model [which corresponds to taking $E(p)=m]$, and as well on the basis of the result of Nelson [10] for a related model, that $H_{n}$ and $\psi_{n}$ should converge in some reasonable sense in $\mathscr{K}$. It has been shown by Sloan [12] that when $d=2$ the vectors $\psi_{n}$ lie, in fact, in a norm compact subset of $\mathscr{F}_{0}$ and that there is a subsequence $n_{j}$ such that $\psi_{n_{j}}$ converges in norm while $H_{n_{j}}$ converges in the sense of generalized strong convergence [9, Chapter 8] to a semibounded self-adjoint operator $H$ on $\mathscr{K}$. His methods do not involve the use of dressing transformations and are therefore quite distinct from those of [10].

When $d \geqq 3$ perturbation theory indicates that $\psi_{n}$ converges weakly to zero. Thus in three or more space dimensions, the model, in all likelihood, exhibits infinite polaron field strength renormalization, and we shall assume this in the following discussion. The weak convergence of $\psi_{n}$ to zero is sometimes described by saying that $\psi_{n}$ moves out of the Fock space $\mathscr{F}_{0}$ and into the physical Hilbert space $\mathscr{K}_{0}$ which is orthogonal to $\mathscr{F}_{0}$. This can be made meaningful in such a way as to give meaning 
to $\lim H_{n}(0)$ as an operator on $\mathscr{K}_{0}$ while at the same time allowing the physical fields to be defined. Let $\mathscr{A}_{0}$ be the algebra generated by the unitary operators

$$
\exp \left(i \int_{E_{d}}\left(\overline{g(k)} b(k)+g(k) b(k)^{*}\right) d k\right)
$$

where $b(k)$ is the pointwise annihilation operator on the zero momentum space $\mathscr{F}_{0}$ (which may be identified with $\mathscr{F}$ ) and $g$ is a square integrable function with compact support. We shall show that for each operator $A$ in $\mathscr{A}_{0}$ there exists a constant $c(A)$ such that

$$
\left\|H_{n}(0) A \psi_{n}\right\| \leqq c(A) \quad \text { for all } n \text {. }
$$

Thus the vectors $A \psi_{n}$ change with $n$ so as always to be located in the "well behaved" part of the domain of $H_{n}(0)$ independently of $n$. We wish to include the (informal) limits of the weakly zero-convergent vectors $A \psi_{n}$ in $\mathscr{K}_{0}$ also since this will allow $\mathscr{A}_{0}$ to act on $\mathscr{K}_{0}$ and $\mathscr{A}_{0}$ largely determines the physical fields. If one takes for the norm of "lim" $A \psi_{n}$ the natural one, namely $\lim \left\|A \psi_{n}\right\|$, then the reader will recognize that meaning can be given to $\mathscr{K}_{0}$ by simply defining it as (the completion of) $\mathscr{A}_{0}$ itself modulo the kernel of the semi-norm $\|A\|_{0}=\lim \left\|A \psi_{n}\right\|$, if this limit exists.

The formalization of the preceding heuristic discussion underlies much of the recent progress in the construction of interacting quantum fields, c.f. [6]. To make this discussion more precise, for any bounded open set $S \subset E_{d}$ let $\mathscr{A}(S)$ be the von Neumann algebra generated by the operators (1.6) where $g$ has support in $S$. Let $\mathscr{A}$ be the closure in norm of the union of these algebras. Put $\omega_{n}(A)=\left(A \psi_{n}, \psi_{n}\right)$ for $A$ in $\mathscr{A}$. There always exists a weak* cluster point of the set $\left\{\omega_{n}\right\}$ of states of $\mathscr{A}$. Choosing one, say $\omega$, we define the zero momentum physical Hilbert space $\mathscr{K}_{0}$ to be the representation space for $\mathscr{A}$ determined by $\omega$ via the GelfandNaimark-Segal construction. Presumably the cluster point $\omega$ is unique but we do not have a proof. If $\sigma$ is the natural representation of $\mathscr{A}$ by operators on $\mathscr{K}_{0}$ and $\psi_{0}$ is the usual cyclic vector for $\sigma(\mathscr{A})$ then $\psi_{0}$ is to be interpreted as the physical rest state of the polaron. The spaces $\mathscr{F}_{p}$ are naturally isomorphic to $\mathscr{F}_{0}$ and, identifying them, the total momentum decomposition of $\mathscr{K}$ may be written $\mathscr{K} \cong L^{2}\left(R_{d}\right) \otimes \mathscr{F}_{0}$ where $\cong$ denotes identification, $R_{d}$ is a copy of $E_{d}$ and a point of $R_{d}$ represents total momentum of polaron plus Boson field rather than merely polaron momentum. We define the physical Hilbert space to be $\mathscr{K}_{p h}=L^{2}\left(R_{d}\right) \otimes \mathscr{K}_{0}$.

In Theorem 1 we show that for each bounded open set $S$ the restrictions $\omega_{n} \mid \mathscr{A}(S)$ form a compact set in norm. This implies that $\omega$ is the limit of a subsequence, that $\mathscr{K}_{0}$ and hence $\mathscr{K}_{p h}$ are separable, and that the restriction of $\sigma$ to $\mathscr{A}(S)$ is unitarily implementable. We say that $\sigma$ is locally Fock in momentum space. The spirit involved in this 
type of result is similar to that of [6] although the techniques of carrying it out are different. Theorem 1 also implies the existence of the time zero Boson fields $\varphi(g)$ and $\pi(g)$ as self-adjoint operators on $\mathscr{K}_{p h}$ for test functions $g$ with compact support in momentum space. In Theorem 2 we show that $\varphi(\cdot)$ and $\pi(\cdot)$ extend by continuity to a larger class of test functions including the Schwartz space $\mathscr{S}$ and in particular smooth functions with compact support in configuration space. In Theorem 3 we construct the total Hamiltonian via the following procedure, which we outline here for the zero momentum subspace only. Define a sequence of sesquilinear forms on $\mathscr{A}_{0}$ by

$$
\langle A, B\rangle_{n}=\left(H_{n}(0) A \psi_{n}, B \psi_{n}\right) .
$$

The inequality (1.7) implies the convergence of some subsequence to a sesquilinear form $\langle$,$\rangle on \mathscr{A}_{0}$. We show that the singular set of this form contains the kernel of the natural injection of $\mathscr{A}_{0}$ into $\mathscr{K}_{0}$. Therefore the form induces a (densely defined) sesquilinear form on $\mathscr{K}_{0}$, which we show to be symmetric, positive definite and separately continuous. The Friedrichs extension theorem now yields a positive self-adjoint operator corresponding to this form. Similar considerations apply to the total space $\mathscr{K}_{p h}$. Since the resulting total Hamiltonian $H$ on $\mathscr{K}_{p h}$ has been obtained as a rather weak kind of limit of the $H_{n}$ there arises the possibility that $H$ is in some sense a trivial operator. In addition to showing that the lower bound of $H$ is $m_{0}$ we show in Theorem 3 that $H$ is actually a rather strong operator by showing that it dominates the local (in momentum space) number operators in the first order sense. I.e., $N_{S} \leqq C_{1} H(0)+C_{2}$ as forms. There is strong evidence that higher order estimates also hold.

In Section 2 we state the main results of the paper. In Section 3 we derive the basic inequalities involving the physical rest state. In Section 4 we prove the theorems stated in Section 2. The basic inequalities of Section 3 are all derived as follows. Fixing the cutoff function $f$, and suppressing the dependence of the Hamiltonian on it, we have $H(p) \geqq m_{0}\left[8\right.$, Theorem 8] for all $p$ in $R_{d}$ and $H(0) \psi=m_{0} \psi$ where $\psi$ is the rest state. Thus for any reasonable operator $A$ we have

$$
\begin{aligned}
(A H(0) \psi, A \psi) & =m_{0}(A \psi, A \psi) \\
& \leqq(H(p) A \psi, A \psi) .
\end{aligned}
$$

Thus $((H(p) A-A H(0)) \psi, A \psi) \geqq 0$. All of the basic inequalities are obtained from this by specializing $A$ in a variety of ways and picking $p$ appropriately.

The present model is in a class of models called persistent interactions because the physical vacuum and bare vacuum coincide. A wide 
class of persistent interactions have been studied by Eckmann [15] and by Albeverio $[13,14]$. The models they study are less singular than the present one and do not require a change of Hilbert space. Their methods are disjoint from the methods used here, partly for this reason. Moreover, whereas, in [15] the mass renormalization term is determined by second order perturbation theory up to a finite quantity, in the present model the mass renormalization must be determined indirectly. This is the case whether the mass renormalization in the present model is accomplished as in the body of this paper or as in Remark 5.4. While the main technique in [15] is a resolvent expansion the main technique in the present paper is the variational approach sketched above. J. Fröhlich has kindly informed me of related work on persisent interactions recently carried out by him in [16].

The work of Sloan [12] was based on the inequalities in an early version of this paper. In turn his work influenced greatly the present form of this paper. In addition to the influence of Sloan's doctoral dissertation I would like to acknowledge helpful discussions with W. Faris and R. Lavine of the Cornell Mathematics Department and with T. Kinoshita and K. Wilson of the Cornell Physics Department. It is a pleasure to thank D. R. Yennie of the Cornell Physics Department for many illuminating and patient discussions of renormalization theory.

\section{Statement of Results}

We denote by $\mathscr{F}$ the Fock space over $L^{2}\left(E_{d}, d k\right)$ where $d k$ is Lebesgue measure on $E_{d}$. Specifically, we regard $\mathscr{F}$ as a space of sequences, $u=\left\{u_{n}\right\}_{n=0}^{\infty}$, of functions, where $u_{n}\left(k_{1}, \ldots, k_{n}\right)$ is a symmetric function on $\left(E_{d}\right)^{n}$ which is in $L^{2}\left(\left(E_{d}\right)^{n}, d k_{1} \ldots d k_{n}\right), u_{0}$ is a complex number, and $\|u\|^{2}=\sum_{n=0}^{\infty}\left\|u_{n}\right\|^{2}<\infty$.

The state space for a single polaron is $L^{2}\left(E_{d}\right)$ and the state space $\mathscr{K}$ for a single polaron plus Boson field is given by (1.5). In the usual way we identify $\mathscr{K}$ with a space of sequences $\psi=\left\{\psi_{n}\right\}_{n=0}^{\infty}$ where $\psi_{n}=\psi_{n}\left(q ; k_{1}, \ldots, k_{n}\right)$ is in $L^{2}\left(\left(E_{d}\right)^{n+1}, d q d k_{1} \ldots d k_{n}\right)$. $\mathscr{E}_{1}$ will denote the manifold in $\mathscr{K}$ consisting of those sequences $\psi$ such that $\psi_{n}=0$ for large $n$ and $\psi_{n}$ is in the Schwartz space $\mathscr{S}\left(\left(E_{d}\right)^{n+1}\right)$ of rapidly decreasing infinitely differentiable complex valued functions on $\left(E_{d}\right)^{n+1}$ for all $n$.

For each point $k$ in $E_{d}$ let $a(k)$ denote the Boson annihilation operator defined on $\mathscr{E}_{1}$. Specifically $(a(k) \psi)_{n}\left(q ; k_{1}, \ldots, k_{n}\right)=(n+1)^{1 / 2} \psi_{n+1}\left(q ; k, k_{1}\right.$, $\left.\ldots, k_{n}\right)$. The polaron annihilation operator $\Psi(q)$, defined by $(\Psi(q) \psi)_{n}$ $\cdot\left(k_{1}, \ldots, k_{n}\right)=\psi_{n}\left(q ; k_{1}, \ldots, k_{n}\right)$, is a linear map from $\mathscr{E}_{1}$ into $\mathscr{F}$. We follow the conventions of [7] in regarding formal products $a^{*}\left(k_{1}\right) \ldots a^{*}\left(k_{j}\right) a\left(k_{j+1}\right) \ldots$ $a\left(k_{n}\right)$ as bilinear forms on $\mathscr{E}_{1} \times \mathscr{E}_{1}$ and integrals of such products as 
integrals of bilinear forms. Moreover when such a bilinear form is the bilinear form of a closed operator with core $\mathscr{E}_{1}$ we shall simply denote the closed operator as the integral of these products as in Eqs. (1.3) and (1.4) above.

As noted in the introduction we take the kinetic energy function $E(q)$ of the polaron to be given by (1.1) where $m$ is a real constant, the bare mass of the polaron. The free Hamiltonian $H_{0}$ of the polaron-Boson system is the self-adjoint operator on $\mathscr{K}$ given by (1.3).

By a cutoff function we shall mean a real valued infinitely differentiable function $f$ on $E_{d}$ with compact support such that $f(k)=f(-k)$. We take the interaction Hamiltonian with momentum cutoff function $f$ to be the self-adjoint operator $H_{I}(f)$ given by (1.4).

The total Hamiltonian for the combined system is the operator $H_{f}$ given by (1.2) which is known [10] to be self-adjoint on its domain.

The total momentum operator $M$ is given by

$$
M_{j}=\text { closure of }\left\{\int_{E_{d}} \Psi^{*}(q) q_{j} \Psi(q) d q+\int_{E_{d}} a(k)^{*} k_{j} a(k) d k\right\} .
$$

$M$ is a $d$-tuple of commuting self-adjoint operators whose spectral projections commute with $H_{f} . M$ thus decomposes $\mathscr{K}$ into a direct integral decomposition

$$
\mathscr{K}=\int_{R_{d}} \mathscr{F}_{p} d p
$$

which reduces $H_{f}$, [8, Propositions 6 and 7], where $R_{d}$ is a copy of $E_{d}$. The infinitesimal subspaces $\mathscr{F}_{p}$ are isomorphic to $\mathscr{F}$ in a natural way and upon identifying $\mathscr{F}_{p}$ with $\mathscr{F}$ for all $p$ we may write (2.2) as

$$
\mathscr{K}=L^{2}\left(R_{d}\right) \otimes \mathscr{F} .
$$

We note that this tensor product decomposition of $\mathscr{K}$ is distinct from that given in Eq. (1.5). In Eq. (1.5) a point $q$ in $E_{d}$ denotes polaron momentum while in Eq. (2.3) a point $p$ in $R_{d}$ has the interpretation of total momentum for the polaron plus Boson field. Properly speaking $\mathscr{K}$ is not equal to the right side of Eq. (2.3) but is unitarily equivalent to it in such a way that the total momentum operator $M_{j}$ on $\mathscr{K}$ corresponds to multiplication by the coordinate function $p_{j}$ in the first factor on the right of $(2.3)$ while the total Hamiltonian corresponds to multiplication by an operator valued function $H(p)$, to be described presently. We use $R_{d}$ to denote total momentum space (of polaron plus Boson field) to reduce confusion of the two distinct tensor product decompositions of $\mathscr{K},(1.5)$ and (2.3).

Moreover we shall write an operator on $\mathscr{K}$ which in the momentum decomposition (2.3) of $\mathscr{K}$ decomposes as a product, $A \otimes B$, as $A \otimes_{m} B$. Here $A$ acts on $L^{2}\left(R_{d}\right)$ while $B$ acts on $\mathscr{F}$. 
Let $\mathscr{E}$ denote the manifold in $\mathscr{F}$ consisting of those sequences $u=\left\{u_{n}\right\}_{n=0}^{\infty}$ such that $u_{n}=0$ for sufficiently large $n$ and $u_{n}$ is in $\mathscr{S}\left(\left(E_{d}\right)^{n}\right)$ for all $n$. For such $u$ define $(b(k) u)_{m}\left(k_{1}, \ldots, k_{n}\right)=(n+1)^{1 / 2} u_{n+1}\left(k, k_{1}, \ldots, k_{n}\right)$. Upon identifying $\mathscr{F}_{p}$ with $\mathscr{F}$ the reduced Hamiltonian $H(p)$ i.e. the restriction of $H_{f}$ to the infinitesimal subspace $\mathscr{F}_{p}$, may be written [8, Proposition 7],

where

$$
H(p)=E(p-P)+\int_{E_{d}} b(k)^{*} \mu(k) b(k) d k+V
$$

$$
P_{j}=\int_{E_{d}} b(k)^{*} k_{j} b(k) d k, \quad j=1, \ldots, d
$$

is the restriction of the Boson momentum operator to $\mathscr{F}_{p}$ and

$$
V=\int_{E_{d}}\left(b(k)+b^{*}(k)\right) f(k) d k .
$$

We remark that relative to the decomposition (2.3) the algebraic tensor product $I \otimes_{m} b(k)$ has a natural extension to the domain $\mathscr{E}_{1}$ and on this domain it is related to $a(k)$ via the equation

$$
I \otimes_{m} b(k)=\left(\int_{E_{d}} \Psi^{*}(q+k) \Psi(q) d q\right) a(k)
$$

The factor in parenthesis is a unitary operator.

Proposition 2.1. Let $m_{0} \geqq 0$. There exists a unique nonnegative real number $m$ depending on the cutoff function $f$ such that

$$
\inf \left(\operatorname{spectrum} H_{f}\right)=m_{0}
$$

when the polaron bare mass is taken to be $m$.

Proof. Suppressing $f$ we observe that, for two different values, $m$ and $m^{\prime}$, of the polaron bare mass, the corresponding total Hamiltonians $H^{m}$ and $H^{m^{\prime}}$ have the same domain and the difference $H^{m}-H^{m^{\prime}}$ is the restriction of the bounded operator

$$
\int \Psi^{*}(q)\left\{\left(m^{2}+|q|^{2}\right)^{1 / 2}-\left(m^{\prime 2}+|q|^{2}\right)^{1 / 2}\right\} \Psi(q) d q
$$

to the domain of $H^{m}$. Since

$$
\left|\left(m^{2}+|q|^{2}\right)^{1 / 2}-\left(m^{\prime 2}+|q|^{2}\right)^{1 / 2}\right| \leqq\left|m-m^{\prime}\right|
$$

we have $\left\|H^{m}-H^{m^{\prime}}\right\| \leqq\left|m-m^{\prime}\right|$. It follows that if $s(m)=\inf$ spectrum $H^{m}$ $=\inf \left\{\left(H^{m} x, x\right) ;\|x\|=1, x \in \mathscr{D}\left(H^{m}\right)\right\}$, then $s(m)$ is a continuous function of $m$. It was shown in [8, Corollary 8.1] that $s(m)=\inf$ spectrum $H^{m}(0)$ and that there is a unit vector $\psi_{m}$ in $\mathscr{F}$ such that $H^{m}(0) \psi_{m}=s(m) \psi_{m}$. 
If $m>m^{\prime}$ then

$$
\begin{aligned}
\left(H^{m^{\prime}}(0) \psi_{m}, \psi_{m}\right)= & \left(H^{m}(0) \psi_{m}, \psi_{m}\right) \\
& -\left(\left(\left(m^{2}+P^{2}\right)^{1 / 2}-\left(m^{\prime 2}+P^{2}\right)^{1 / 2}\right) \psi_{m}, \psi_{m}\right)<s(m) .
\end{aligned}
$$

Hence $s$ is strictly increasing. Since $\int \mu(k) b(k)^{*} b(k) d k+V$ is bounded below [10] it follows from (2.4) with $p=0$ that $s(m)$ increases to $\infty$ as $m$ increases to $\infty$. Now $s(m)=m$ if $f=0$ with inf spectrum $H^{m}(0)$ achieved at the bare rest state $\Omega$ in $\mathscr{F}$. Since $(V \Omega, \Omega)=0$ it follows that for arbitrary $f$ in the class considered $s(m) \leqq m$. Hence the equation $s(m)=m_{0}$ has a unique positive solution.

Henceforth we shall assume that the bare mass $m$ is chosen so that $\inf \left(\right.$ spectrum $H_{f}$ ) remains fixed at the physical mass $m_{0}$ of the polaron. Thus $m$ will vary with $f$ and will in fact approach $+\infty$ as the cutoff function $f$ is allowed to approach $\lambda \mu(k)^{-1 / 2}$.

For each complex valued function $g$ in $L^{2}\left(E_{d}\right)$ put $R_{0}(g)$ for the selfadjoint operator [2]

$$
R_{0}(g)=\int_{E_{d}}\left(\overline{g(k)} b(k)+g(k) b(k)^{*}\right) d k .
$$

For each bounded open set $B$ in momentum space we denote by $\mathscr{A}(B)$ the von Neumann algebra generated by the operators $\exp \left(i R_{0}(g)\right)$ where $g$ runs over the functions in $L^{2}\left(E_{d}\right)$ with support in $B$. Let $\mathscr{A}$ be the closure in norm of the union $\cup \mathscr{A}(B)$ where the union is over all bounded open sets $B \subset E_{d}$.

Each cutoff function $f$ determines a state $\omega_{f}$ of the $C^{*}$ algebra $\mathscr{A}$ as follows. $\mathscr{A}$ is to be regarded as an algebra of operators on the zero momentum subspace $\mathscr{F}_{0}$. Let $\psi_{f}$ be a unit eigenvector of $H_{f}(0)$ in $\mathscr{F}_{0}$ with eigenvalue $m_{0}$. The existence and uniqueness of $\psi_{f}$ was shown in [8, Theorem 8]. Define

$$
\omega_{f}(A)=\left(A \psi_{f}, \psi_{f}\right), \quad A \in \mathscr{A} .
$$

Theorem 1. Let $f_{n}$ be a sequence of cutoff functions such that for each bounded open set $B$ in $E_{d}$ the sequence

$$
\int_{B}\left|f_{n}(k)\right|^{2} d k+\int_{B}\left|\nabla f_{n}(k)\right|^{2} d k
$$

is bounded, where $\nabla f$ denotes the gradient of $f$. Then for each bounded open set $B$ the restrictions of the corresponding states $\omega_{n}$ to $\mathscr{A}(B)$ form a relatively compact set in the norm topology of the dual space $\mathscr{A}(B)^{*}$.

Now let $\lambda$ be a real constant and let $f_{n}$ be a sequence of cutoff functions which converge uniformly on bounded sets in $E_{d}$ to $\lambda \mu(k)^{-1 / 2}$. We may and shall choose the $f_{n}$ so that the gradients $\nabla f_{n}$ satisfy the boundedness condition of Theorem 1 . For example we may choose $f_{n}$ to be any 
cutoff function which is equal to $\lambda \mu(k)^{-1 / 2}$ when $|k| \leqq n$ and equal to zero if $|k| \geqq n+1$. The sequences $f_{n}$ and $\left|\nabla f_{n}\right|$ can both be chosen to be uniformly bounded. Although we should think of this sequence as being the typical sequence to which the following theorems apply none of the results actually depend on such a strong sense of convergence of $f_{n}$ to $\lambda \mu(k)^{-1 / 2}$ and we shall state in each case just what we actually require of the sequence $f_{n}$.

If $\omega_{n}$ denotes the corresponding state on $\mathscr{A}$ then since the set of states on $\mathscr{A}$ is weakly compact in the dual space $\mathscr{A}^{*}$ there exists a weak accumulation point $\omega$ of the sequence $\omega_{n}$. That is, there exists a state $\omega$ which lies in the weak* closure of the set $\left\{\omega_{m}, \omega_{n+1}, \ldots\right\}$ for all $n$. We do not know at the present time whether the state $\omega$ is unique i.e. whether there is just one such accumulation point. In fact none of the following results actually depend crucially on the convergence of the functions $f_{n}$ but merely (e.g. in Theorem 2 ) on a slight strengthening of the hypothesis of Theorem 1. (The hypothesis of Theorem 1 already implies the existence of a subsequence of the functions $f_{n}$ which is convergent locally in $L^{2}$.) In the following corollaries we consider any such limit state $\omega . \omega$ gives rise in a well known manner to a Hilbert space $\mathscr{K}_{0}$, a representation $\sigma$ of $\mathscr{A}$ on $\mathscr{K}_{0}$, and a unit vector $\psi_{0}$ in $\mathscr{K}_{0}$ which is cyclic for $\sigma(\mathscr{A})$ and such that $\omega(A)=\left(\sigma(A) \psi_{0}, \psi_{0}\right)$. As noted in the introduction $\mathscr{K}_{0}$ is to be interpreted as the physical one polaron Hilbert space of total momentum zero and $\psi_{0}$ is the physical rest state of the polaron. We define the full physical Hilbert space for a single polaron plus field as

$$
\mathscr{K}_{p h}=L^{2}\left(R_{d}\right) \otimes \mathscr{K}_{0} .
$$

Corollary 1.1. Under the same hypotheses as in Theorem 1 there exists, for any weak accumulation point $\omega$, a subsequence $\omega_{n_{j}}$ such that

i) $\omega_{n_{j}}(A)$ converges to $\omega(A)$ for every operator $A$ in $\mathscr{A}$.

ii) The restrictions $\omega_{n_{j}} \mid \mathscr{A}(B)$ converge in norm to $\omega \mid \mathscr{A}(B)$ for every bounded open set $B \subset E_{d}$.

For each bounded open set $B$ the restriction $\sigma \mid \mathscr{A}(B)$ is an isomorphism which is continuous in the strong operator topologies. In fact there is a (non-unique) unitary operator $U_{B}$ from $\mathscr{F}$ onto $\mathscr{K}_{0}$ which implements $\sigma \mid \mathscr{A}(B)$. Furthermore $\mathscr{K}_{0}$ is separable.

In keeping with current terminology the unitary implementability of $\sigma \mid \mathscr{A}(B)$ may be referred to by saying that the representation $\sigma$ is locally Fock in momentum space.

For each bounded open set $B$ in $E_{d}$ let $\mathscr{C}(B)$ be the von Neumann algebra on $\mathscr{K}$ generated by the operators $C \otimes_{m} A$ relative to the total momentum decomposition (2.3) where $C$ runs over all bounded operators on $L^{2}\left(R_{d}\right)$ and $A$ is in $\mathscr{A}(B)$. We put $\mathscr{C}$ for the closure in norm of $\cup \mathscr{C}(B)$ where the union is taken over all bounded open sets $B$ in $E_{d}$. 
We note that $\mathscr{C}$ contains all observables corresponding to bounded functions of the total momentum of polaron plus field.

Corollary 1.2. Under the same hypotheses as in Theorem 1, there exists, for any weak accumulation point $\omega$, a unique * isomorphism $\hat{\sigma}$ from $\mathscr{C}$ to bounded operators on $\mathscr{K}_{p h}$ with the following two properties.

a) For any bounded operator $C$ on $L^{2}\left(R_{d}\right)$ and any operator $A$ in $\mathscr{A}$ there holds

$$
\hat{\sigma}\left(C \otimes_{m} A\right)=C \otimes \sigma(A)
$$

b) For any bounded open set $B$ in $E_{d}$ the restriction of $\hat{\sigma}$ to the unit ball of $\mathscr{C}(B)$ is continuous in the strong operator topology to the weak operator topology. Moreover

c) the restriction of $\hat{\sigma}$ to $\mathscr{C}(B)$ is unitarily implementable for any bounded open set $B$ and is therefore continuous in the strong operator topologies.

We wish next to show that the Boson time-zero physical fields $\varphi(u)$ and $\pi(u)$ are definable on $\mathscr{K}_{p h}$ for a reasonably large class of test functions $u$. The bare time-zero Boson field operators are defined on $\mathscr{K}$ by

and

$$
\varphi(u)=\int_{E_{d}}\left(\hat{u}(k) a(k)+\overline{\hat{u}(k)} a(k)^{*}\right) \mu(k)^{-1 / 2} d k
$$

$$
\pi(u)=i^{-1} \int_{E_{d}}\left(\hat{u}(k) a(k)-\overline{\hat{u}(k)} a(k)^{*}\right) \mu(k)^{1 / 2} d k
$$

where $u$ is a real valued test function on configuration space and $\hat{u}$ is its Fourier transform. These bare fields $\varphi$ and $\pi$ are well defined self-adjoint operators on $\mathscr{K}$ if $\hat{u}(k) \mu(k)^{1 / 2}$ is in $L^{2}\left(E_{d}, d k\right)$. We shall first show that the physical field versions of $\varphi$ and $\pi$ are definable on $\mathscr{K}_{p h}$ when $\hat{u}$ has compact support in momentum space. This is a consequence of Corollary 1.2 and Proposition 2.2 below. Such functions $u$ cannot have compact support in configuration space, however, and the objective of Theorem 2 will be to extend the physical fields so as to be defined for a test function space large enough to contain at least the Schwartz space of real valued rapidly decreasing smooth functions on configuration space.

It will be convenient to deal with the fields $\varphi$ and $\pi$ simultaneously by studying instead the self-adjoint operator $R(g)$ on $\mathscr{K}$ defined for any complex valued function $g$ in $L^{2}\left(E_{d}, d k\right)$ by

$$
R(g)=\int_{E_{d}}\left\{(\overline{g(k)}) a(k)+g(k) a(k)^{*}\right\} d k .
$$

If $\overline{g(k)}=\hat{u}(k) \mu(k)^{-1 / 2}$ then $R(g)=\varphi(u)$ and if $\overline{g(k)}=-i \hat{u}(k) \mu(k)^{1 / 2}$ then $R(g)=\pi(u)$. While the operators $R(g)$ act on the bare one polaron space $\mathscr{K}$, the time-zero physical fields are operators on $\mathscr{K}_{p h}$. The physical field 
version of $R(g)$ is denoted in Theorem 2 by $S(g)$. The physical $\varphi$ and $\pi$ fields may be recovered from $S$ in the same way that the bare $\varphi$ and $\pi$ fields were recovered from $R$.

Proposition 2.2. Let $B$ be a bounded open set in $E_{d}$. If $g$ is a complex valued function in $L^{2}\left(E_{d}\right)$ with support in $B$ then $e^{i R(g)}$ lies in $\mathscr{C}(B)$. If $h$ is a bounded real valued measurable function on $E_{d}$ with support in $B$ then the self-adjoint operator $D_{h}$ on $\mathscr{K}$ defined by

$$
D_{h}=\int_{E_{d}} h(k) a(k)^{*} a(k) d k
$$

has the property that $e^{i t D_{h}}$ is in $\mathscr{C}(B)$ for all real $t$.

Remark. In view of Corollary 1.2 and Proposition 2.2 we can now define the physical fields $S(g)$ by the equation $e^{i t S(g)}=\hat{\sigma}\left(e^{i t R(g)}\right)$ for all real $t$, provided $g$ has compact support in momentum space.

Theorem 2. Let $\omega$ be any weak accumulation point of the $\omega_{n}$ and let $\mathscr{K}_{0}$ and $\mathscr{K}_{p h}$ be as above. Let $\tau$ be a non-negative real number such that

$$
\int_{E_{d}} \mu(k)^{-\tau}\left(\mu(k)^{-1 / 2} / \mu(k)\right)^{2} d k<\infty .
$$

Assume that the cutoff functions $f_{n}$ are so chosen that $\left|f_{n}(k)\right| \leqq$ const $\mu(k)^{-1 / 2}$ and such that the hypothesis of Theorem 1 is satisfied.

a) Let $K_{\tau}=L^{2}\left(E_{d}, \mu(k)^{\tau} d k\right)$. There exists a unique map $g \rightarrow S(g)$ from $K_{\tau}$ to self-adjoint operators on $\mathscr{K}_{p h}$ such that the following two properties hold.

i) $e^{i t S(g)}$ is a strongly continuous function of $g$ in $K_{\tau}$ norm for each real number $t$.

ii) $\hat{\sigma}\left(e^{i t R(g)}\right)=e^{i t S(g)}$ for all real $t$ and all $g$ in $K_{\tau}$ with compact support.

Moreover $S(g)$ satisfies the Heisenberg commutation relations in the bounded form of Weyl:

$$
e^{i S(g)} e^{i S\left(g^{\prime}\right)}=e^{i \operatorname{Im}\left(g, g^{\prime}\right)} e^{i S\left(g+g^{\prime}\right)}
$$

which is valid for $g$ and $g^{\prime}$ in $K_{\tau}$ where $\left(g, g^{\prime}\right)=\int g(k) \overline{g(k)^{\prime}} d k$.

b) Let $L_{\tau}$ be the set of all real valued measurable functions $h$ on $E_{d}$ such that

$$
\|h\|_{L_{\tau}}=\sup _{k} \mu(k)^{\tau}|h(k)|<\infty .
$$

There exists a unique map $h \rightarrow N(h)$ from $L_{\tau}$ to self-adjoint operators on $\mathscr{K}_{p h}$ such that the following two properties hold.

iii) for each real number $t e^{i t N(h)}$ is a strongly continuous function of $h$ on $L_{\tau}$-bounded sets in the topology of pointwise convergence of sequences. (i.e. if $\left\|h_{n}\right\|_{L_{\tau}} \leqq$ constant and $h_{n}(k) \rightarrow h(k)$ for each $k$ then $e^{i t N\left(h_{n}\right)}$ converges strongly to $e^{i t(h)}$ for each real t.) 
iv) $\hat{\sigma}\left(e^{i t D_{h}}\right)=e^{i t N(h)}$, whenever $h$ is a real bounded measurable function with compact support, where $D_{h}$ is given by (2.13).

Moreover $N(h)$ is order preserving on $L_{\tau}$. The operators $N(h), h$ in $L_{\tau}$, are mutually commuting, and $N\left(\alpha h+\beta h^{\prime}\right)=$ closure of $\left(\alpha N(h)+\beta N\left(h^{\prime}\right)\right)$ for $\alpha$ and $\beta$ real and $h$ and $h^{\prime}$ in $L_{\tau}$. Finally

$$
e^{i N(h)} S(g) e^{-i N(h)}=S\left(e^{i h} g\right)
$$

for all $h$ in $L_{\tau}$ and $g$ in $K_{\tau}$.

Corollary 2.1. For any function $u$ in $L^{2}\left(R_{d}\right)$, and $h$ in $L_{\tau}, u \otimes \psi_{0}$ is in the domain of $N(h)$, where $\psi_{0}$ is the physical rest state.

Denote by $\mathscr{A}_{0}$ the set of all finite linear combinations of operators on $\mathscr{F}$ of the form $e^{i R_{0}(g)}$ where $g$ is in $L^{2}\left(E_{d}\right), g$ has compact support, and $R_{0}(g)$ is given by (2.6). Clearly $\mathscr{A}_{0}$ is a subalgebra of $\mathscr{A}$ and is irreducible on $\mathscr{F}$. In particular, we note that for any rest state $\psi_{n}, \mathscr{A}_{0} \psi_{n}$ is dense in $\mathscr{F}$.

Theorem 3. Assume the hypotheses and notation of Theorem 2. Let $\omega$ be a weak limit point of the $\omega_{n}$. There exists a subsequence $\omega_{n_{j}}$ satisfying the conclusion of Corollary 1.1 and a self-adjoint operator $H$ on $\mathscr{K}_{p h}$ having the following three properties.

i) $\lim _{j \rightarrow \infty}\left(H_{n_{j}}\left(u \otimes_{m} A \psi_{n_{j}}\right), v \otimes_{m} B \psi_{n_{j}}\right)=\left(H\left(u \otimes \sigma(A) \psi_{0}\right), v \otimes \sigma(B) \psi_{0}\right)$

for all $A$ and $B$ in $\mathscr{A}_{0}$ and all $u$ and $v$ in $C_{c}\left(R_{d}\right)$

ii) inf spectrum $H=m_{0}$.

iii) Let $h$ be a non-negative measurable function in $L_{\tau}[c$.f. Theorem 2, part b)]. Assume that $h$ is even, i.e. $h(-k)=h(k)$, and that for all $k$ in $E_{d} \mu(k)-h(k) \geqq c>0$ for some positive constant $c$. Let $N(h)$ be the number like operator on $\mathscr{K}_{p h}$ described in Theorem 2. Assume, in addition to the preceding hypotheses, that $f_{n}(k)$ converges to $\lambda \mu(k)^{-1 / 2}$ for each $k$, where $\lambda$ is a non-zero real number. Let

$$
d(h)=\int_{E_{d}} h(k)\left[\frac{\lambda \mu(k)^{-1 / 2}}{\mu(k)-h(k)}\right]^{2} d k
$$

For any number a with $0<a$ we write $L^{2}\left(S_{a}\right)$ for the subspace of $L^{2}\left(R_{d}\right)$ consisting of functions $g$ such that $g(k)=0$ if $|k|>a$. The subspace $L^{2}\left(S_{a}\right) \otimes \mathscr{K}_{0}$ of $\mathscr{K}_{p h}$ is invariant under $N(h)$ and $H$, and in this subspace there holds

$$
\left\|N(h)^{1 / 2} \psi\right\| \leqq\left\|(H+d(h)+a)^{1 / 2} \psi\right\|, \quad \psi \in\left(L^{2}\left(S_{a}\right) \otimes \mathscr{K}_{0}\right) \cap \mathscr{D}\left(H^{1 / 2}\right) .
$$

Remark. If $h$ is taken as the characteristic function of a bounded open symmetric set $B$ (i.e., $-B=B$ ) times a suitable positive constant $\alpha$ 
then $N(h)$ is formally $\alpha$ times the number operator $N_{B}$ associated with the region $B$ and (2.17) reads

$$
\left\|N_{B}^{1 / 2} \psi\right\| \leqq \alpha^{-1 / 2}\left\|(H+d(h)+a)^{1 / 2} \psi\right\|, \quad \psi \in \mathscr{D}\left(H^{1 / 2}\right) \cap\left(L^{2}\left(S_{a}\right) \otimes \mathscr{K}_{0}\right) .
$$

\section{§ 3. The Basic Inequalities}

Throughout this section we consider a fixed cutoff function $f$. We recall that $f$ is a real valued infinitely differentiable function on $E_{d}$ with compact support and satisfying $f(-k)=f(k)$. We write

and

$$
H=H_{f}-m_{0} I
$$

$$
H(p)=H_{f}(p)-m_{0} I .
$$

Then $H$ and $H(0)$ have lower bound zero, because of the choice of the bare mass $m$, and $H(p) \geqq 0$.

Lemma 3.1. For every strictly positive integer $n$ and every non-empty subset $\alpha \subset\{1, \ldots, n\}$ there exists a bounded continuous non-negative function $g_{\alpha}^{(n)}\left(k_{1}, \ldots, k_{n}\right)$ on $\left(E_{d}\right)^{n}$ such that for any vector $\psi$ in $\mathscr{E}$ there holds

$$
\begin{aligned}
\left\|b\left(k_{1}\right) \ldots b\left(k_{n}\right) \psi\right\| \leqq & \left(\prod_{j=1}^{n} \mid \frac{f\left(k_{j}\right)}{\mu\left(k_{j}\right)}\right)\|\psi\| \\
& +\sum_{\alpha} g_{\alpha}^{n}\left(k_{1}, \ldots, k_{n}\right)\|A(\alpha) H(0) \psi\|
\end{aligned}
$$

where the sum runs over all non-empty subsets $\alpha$ of $\{1, \ldots, n\}$ and $A(\alpha)=\prod_{j \in \alpha} b\left(k_{j}\right)$.

Proof. The proof is by induction on $n$. We first consider the case $n=1$. Since $E(-k-P) b(k) \psi=b(k) E(-P) \psi$ for $\psi$ in $\mathscr{E}$ we have

$$
(H(-k) b(k)-b(k) H(0)) \psi=-\mu(k) b(k) \psi-f(k) \psi .
$$

Now $H(-k) \geqq 0$. Consequently, for any vector $\psi$ in $\mathscr{E}$,

$$
\begin{aligned}
0 & \leqq(H(-k) b(k) \psi, b(k) \psi) \\
& =((H(-k) b(k)-b(k) H(0)) \psi, b(k) \psi)+(b(k) H(0) \psi, b(k) \psi) \\
& =-\mu(k)\|b(k) \psi\|^{2}-f(k)(\psi, b(k) \psi)+(b(k) H(0) \psi, b(k) \psi) .
\end{aligned}
$$

Hence

$$
\mu(k)\|b(k) \psi\|^{2} \leqq|f(k)|\|\psi\|\|b(k) \psi\|+\|b(k) H(0) \psi\|\|b(k) \psi\| .
$$

Dividing by $\|b(k) \psi\|$ yields

$$
\|b(k) \psi\| \leqq \frac{|f(k)|}{\mu(k)}\|\psi\|+\frac{1}{\mu(k)}\|b(k) H(0) \psi\| .
$$


This is the inequality (3.1) with $g_{\{1\}}^{(1)}(k)=\mu(k)^{-1}$.

Assume (3.1) is valid for $n$. We show it is valid for $n+1$. Let $k_{1}, \ldots, k_{n+1}$ be $n+1$ points in $E_{d}$. Put $A=\prod_{j=1}^{n+1} b\left(k_{j}\right)$ and $A^{(i)}=\prod_{j \neq i} b\left(k_{j}\right)$. Then $A$ and $A^{(i)}$ take $\mathscr{E}$ into $\mathscr{E}$. Moreover, since

$$
E\left(-k_{1}-\cdots-k_{n+1}-P\right) A-A E(-P)=0 \text { on } \mathscr{E}
$$

we have, for any $\psi$ in $\mathscr{E}$,

Hence

$$
\begin{aligned}
H\left(-k_{1}-\cdots-k_{n}\right) A \psi-A H(0) \psi= & -\left(\sum_{i=1}^{n+1} \mu\left(k_{i}\right)\right) A \psi \\
& -\sum_{i=1}^{n+1} f\left(k_{i}\right) A^{(i)} \psi .
\end{aligned}
$$

$$
\begin{aligned}
0 & \leqq\left(H\left(-k_{1}-\cdots-k_{n+1}\right) A \psi, A \psi\right) \\
& =\left(\left(H\left(-k_{1}-\cdots-k_{n+1}\right) A-A H(0)\right) \psi, A \psi\right)+(A H(0) \psi, A \psi) \\
& =-\left(\sum_{i=1}^{n+1} \mu\left(k_{i}\right)\right)\|A \psi\|^{2}-\sum_{i=1}^{n+1} f\left(k_{i}\right)\left(A^{(i)} \psi, A \psi\right)+(A H(0) \psi, A \psi) .
\end{aligned}
$$

Thus

$$
\begin{aligned}
\left(\sum_{i=1}^{n+1} \mu\left(k_{i}\right)\right)\|A \psi\|^{2} \leqq & \sum_{i=1}^{n+1}\left|f\left(k_{i}\right)\right|\left\|A^{(i)} \psi\right\|\|A \psi\| \\
& +\|A H(0) \psi\|\|A \psi\| .
\end{aligned}
$$

Dividing by $\|A \psi\|$ yields

$$
\left(\sum_{i=1}^{n+1} \mu\left(k_{i}\right)\right)\|A \psi\| \leqq \sum_{i=1}^{n+1}\left|f\left(k_{i}\right)\right|\left\|A^{(i)} \psi\right\|+\|A H(0) \psi\| .
$$

Now apply the induction assumption to $\left\|A^{(i)} \psi\right\|$, yielding

$$
\begin{aligned}
\left(\sum_{i=1}^{n+1} \mu\left(k_{i}\right)\right) & \left.\|A \psi\| \leqq\left(\sum_{i=1}^{n+1}\left|f\left(k_{i}\right)\right| \prod_{j \neq i} \mid \frac{f\left(k_{j}\right)}{\mu\left(k_{j}\right)}\right)\right)\|\psi\| \\
& +\sum_{i=1}^{n+1}\left|f\left(k_{i}\right)\right| \sum_{\alpha \subset\{1, \ldots, \hat{i}, \ldots, n+1\}} g_{\alpha}^{(n)}\left(k_{1}, \ldots, \hat{k}_{i}, \ldots, k_{n+1}\right)\|A(\alpha) H(0) \psi\| \\
& +\|A H(0) \psi\| .
\end{aligned}
$$

Since

$$
\sum_{i=1}^{n+1}\left|f\left(k_{i}\right)\right| \prod_{j \neq i}\left|f\left(k_{j}\right) / \mu\left(k_{j}\right)\right|=\left(\sum_{i=1}^{n+1} \mu\left(k_{i}\right)\right) \prod_{j}\left|f\left(k_{j}\right) / \mu\left(k_{j}\right)\right|
$$


the desired inequality follows with

$$
g_{\alpha}^{(n+1)}\left(k_{1}, \ldots, k_{n+1}\right)=\left(\sum_{i=1}^{n+1} \mu\left(k_{i}\right)\right)^{-1} \sum_{i \notin \alpha}\left|f\left(k_{i}\right)\right| g_{\alpha}^{(n)}\left(k_{1}, \ldots, \hat{k}_{i}, \ldots, k_{n+1}\right)
$$

where the last sum should be replaced by one if $\alpha=\{1, \ldots, n+1\}$, and otherwise the subscript of $g_{\alpha}^{(n)}$ should be regarded as a subset of $\{1, \ldots, \hat{i}, \ldots, n+1\}$.

Remark 3.2. Let $v\left(k_{1}, \ldots, k_{n}\right)$ be a bounded real valued measurable function on $\left(E_{d}\right)^{n}$. We assert that the equation

$$
B_{v}=\int_{\left(E_{d}\right)^{n}} v\left(k_{1}, \ldots, k_{n}\right) b\left(k_{n}\right)^{*} \ldots b\left(k_{1}\right)^{*} b\left(k_{1}\right) \ldots b\left(k_{n}\right) d k_{1} \ldots d k_{n}
$$

defines a self-adjoint operator on $\mathscr{F}$. This means, in accordance with our convention, that the right side is the bilinear form of a self-adjoint operator with core $\mathscr{E}$. In fact one computes readily that the right side is the bilinear form of the operator $B$ on $\mathscr{E}$ whose action on an $r$ vector $\psi$ in $\mathscr{E}$ is

$(B \psi)\left(k_{1}, \ldots, k_{r}\right)=\left\{\begin{array}{lr}r(r-1) \ldots(r-n+1) P_{s} v\left(k_{1}, \ldots, k_{n}\right) \psi\left(k_{1}, \ldots, k_{r}\right) r \geqq n \\ 0 & r<n\end{array}\right.$

where $P_{s}$ is the symmetrization projection. $B$ is clearly a bounded operator on $r$ vectors in $L^{2}$ norm with norm at most $r^{n}\|v\|_{\infty}$. Hence if $\psi=\Sigma \psi_{r}$ is in $\mathscr{E}$ then $\|B \psi\|^{2} \leqq \sum_{r}\|v\|_{\infty}^{2}\left\|N^{n} \psi_{r}\right\|^{2}=\|v\|_{\infty}^{2}\left\|N^{n} \psi\right\|^{2}$ where $N$ is the number operator. Thus every vector $\psi$ in $\mathscr{E}$ is an analytic vector for $B$, which is clearly symmetric, and therefore $\mathscr{E}$ is a core for a unique selfadjoint extension $B_{v}$ of $B$. Since $\|B \psi\| \leqq\|v\|_{\infty}\left\|N^{n} \psi\right\|$ for $\psi$ in $\mathscr{E}$ we have $\mathscr{D}\left(N^{n}\right) \subset \mathscr{D}\left(B_{v}\right)$ and

$$
\left\|B_{v} \psi\right\| \leqq\|v\|_{\infty}\left\|N^{n} \psi\right\| \quad \text { for } \psi \text { in } \mathscr{D}\left(N^{n}\right) .
$$

This remark is closely related to observations made in $[5,7]$. We note also that if $v \geqq 0$ then $B_{v} \geqq 0$.

Proposition 3.3. Let $\psi$ be the physical rest state. I.e., $\psi$ is a unit vector in $\mathscr{F}_{0}$ satisfying $H(0) \psi=0$. Let $v$ be a bounded measurable real valued function on $\left(E_{d}\right)^{n}$ where $n$ is a strictly positive integer. Then $\psi$ is in the domain of $B_{v}$ and

$$
\left|\left(B_{v} \psi, \psi\right)\right| \leqq \int\left|v\left(k_{1}, \ldots, k_{n}\right) \prod_{j=1}^{n}\left(f\left(k_{j}\right) / \mu\left(k_{j}\right)\right)^{2}\right| d k_{1} \ldots d k_{n} .
$$

Proof. Let $K=E(P)+\left(H_{0}\right)_{b}-m_{0}$. Then $H(0)=K+V$. We first show by induction on $j$ that $\psi$ is in $\mathscr{D}\left((N+1)^{j / 2} K\right)$ for all integers $j \geqq 0$ (Sloan [12]). This is so for $j=0$ because $\mathscr{D}(K)=\mathscr{D}(H(0))$. Moreover if it is true for $j=n \geqq 0$ then since $\mathscr{D}\left((N+1)^{j / 2} K\right) \subset \mathscr{D}\left((N+1)^{\frac{j}{2}+1}\right) \subset \mathscr{D}\left((N+1)^{\frac{J}{2}+\frac{1}{2}} V\right)$ 
(the last containment follows from the commutation relations) it follows from the equation $(N+1)^{j / 2} K \psi=-(N+1)^{j / 2} V \psi$ that the left side is in the domain of $(N+1)^{1 / 2}$ since the right side is. This establishes that $\psi \in \mathscr{D}\left((N+1)^{j / 2} K\right)$ for all $j$. Therefore $\psi$ is also in $\mathscr{D}\left((N+1)^{j / 2}(K+1)\right)$ for all $j$.

If $n$ is the strictly positive integer in the statement of the proposition then, since $(N+1)^{n}(K+1)$ is the closure of its restriction to $\mathscr{E}$, there exists a sequence $\psi_{j}$ in $\mathscr{E}$ such that $\psi_{j}$ converges to $\psi$ in norm and $(N+1)^{n}(K+1) \psi_{j}$ converges to $(N+1)^{n}(K+1) \psi$ in norm. Since $\left\|(N+1)^{n} \varphi\right\| \leqq\left\|(N+1)^{n}(K+1) \varphi\right\|$ for all $\varphi$ in $\mathscr{D}\left((N+1)^{n}(K+1)\right)$ it follows from 3.4) that $\psi$ is in $\mathscr{D}\left(B_{v}\right)$ and $B_{v} \psi=\lim _{j} B_{v} \psi_{j}$. Moreover since $\left\|N^{n} V \varphi\right\| \leqq$ const $\left\|(N+1)^{n}(K+1) \varphi\right\|$, as follows from the commutation relations, we may conclude that $N^{n} H(0) \psi_{j}$ converges to $N^{n} H(0) \psi=0$.

Assume first that $v$ is non-negative (and bounded). Apply the inequality (3.1) to $\psi_{j}$. Square both sides of (3.1), multiply by $v\left(k_{1}, \ldots, k_{n}\right)$ and integrate with respect to $k_{1}, \ldots, k_{n}$ over $\left(E_{d}\right)^{n}$ to obtain

$$
\begin{aligned}
& \int v\left(k_{1}, \ldots, k_{n}\right)\left\|b\left(k_{1}\right) \ldots b\left(k_{n}\right) \psi_{j}\right\|^{2} d k_{1} \ldots d k_{n} \\
& \quad \leqq\left(\int v(k) \prod_{i=1}^{n}\left|\frac{f\left(k_{i}\right)}{\mu\left(k_{i}\right)}\right|^{2} d^{n} k\right)\left\|\psi_{j}\right\|^{2}+\sum_{\alpha, \beta} \int d_{\alpha, \beta}(k) D_{\alpha} D_{\beta} d k
\end{aligned}
$$

where $k=\left(k_{1}, \ldots, k_{n}\right)$, the sum on $\alpha$ and $\beta$ runs over the collection of all pairs of subsets of $\{1, \ldots, n\}$ for which at least one of the sets $\alpha, \beta$ is non-empty, $D_{\alpha}=\left\|A(\alpha) H(0) \psi_{j}\right\|$ if $\alpha$ is not empty, $D_{\alpha}=\left\|\psi_{j}\right\|$ is $\alpha$ is empty, and $d_{\alpha, \beta}(k)$ is a bounded non-negative measurable function which, if $\alpha$ or $\beta$ is empty, has compact support in $\left(E_{d}\right)^{n}$. We assert that the finite sum $\sum_{\alpha, \beta} \ldots$ goes to zero as $j \rightarrow \infty$. In fact

$$
\left|\int d_{\alpha, \beta}(k) D_{\alpha} D_{\beta} d k\right|^{2} \leqq\left(\int\left|d_{\alpha, \beta}(k)\right| D_{\alpha}^{2} d k\right)\left(\int\left|d_{\alpha, \beta}(k)\right| D_{\beta}^{2} d k\right) .
$$

At least one of the two integrals on the right (corresponding to a nonempty subscript) is of the form $\left(B H(0) \psi_{j}, H(0) \psi_{j}\right)$ where $B$ is an operator of the type described in Remark 3.2 of order less than or equal to $n$. Hence one of these two integrals is dominated by const. $\left\|N^{n} H(0) \psi_{j}\right\|$ $\left\|H(0) \psi_{j}\right\|$ which goes to zero as $j \rightarrow \infty$. The other one is clearly bounded or also goes to zero.

The left side of (3.6) is $\left(B_{v} \psi_{j}, \psi_{j}\right)$ which converges to $\left(B_{v} \psi, \psi\right)$. This concludes the proof in case $v \geqq 0$. In the general case write $v=v_{1}-v_{2}$ where $v_{1}$ and $v_{2}$ are non-negative and have disjoint support. Then $|v|=v_{1}+v_{2}$. Since $B_{v} \psi=\lim B_{v} \psi_{j}=\lim \left(B_{v_{1}} \psi_{j}-B_{v_{2}} \psi_{j}\right)=B_{v_{1}} \psi-B_{v_{2}} \psi$ we have $\left|\left(B_{v} \psi, \psi\right)\right|=\left|\left(B_{v_{1}} \psi, \psi\right)-\left(B_{v_{2}} \psi, \psi\right)\right| \leqq\left(B_{v_{1}} \psi, \psi\right)+\left(B_{v_{2}} \psi, \psi\right)=\left(B_{|v|} \psi, \psi\right)$ which is dominated by the right side of (3.5), as we have seen. This concludes the proof. 
Remark 3.4. The preceding proposition implies that in the Schrödinger representation the rest state $\psi$ is infinitely differentiable in all directions in the $L^{2}$ sense. The next corollary will not be used in an essential way in this paper but we include it here since it belongs here logically and we anticipate its usefulness in future developments of this theory. It can be used to show that in the Schrödinger representation the physical rest state $\psi$ is an analytic function (in a sense which we shall not make precise here).

Corollary 3.5. Let $h$ be a bounded, non-negative, measurable function on $E_{d}$. Let $N_{h}$ be the non-negative, self-adjoint operator on $\mathscr{F}$ given by

$$
N_{h}=\int_{E_{d}} h(k) b(k)^{*} b(k) d k .
$$

The physical rest state $\psi$ (of unit norm) is in the domain of $e^{N_{h}}$ and

$$
\left(e^{N_{h}} \psi, \psi\right) \leqq c(h)
$$

where

$$
c(h)=\exp \left\{\int_{E_{d}}\left(\left(e^{h(k)}-1\right) f(k)^{2} / \mu(k)^{2}\right) d k\right\} .
$$

Proof. If $z_{1}, \ldots, z_{r}$ are any real numbers then $\prod_{j=1}^{r} z_{j}=\prod_{j=1}^{r}\left(1+\left(z_{j}-1\right)\right)$ $=\sum_{\alpha} \prod_{j \in \alpha_{\alpha}}\left(z_{j}-1\right)$ where $\alpha$ runs over all subsets of $\{1, \ldots, r\}$ and an empty product is interpreted as 1 . Thus if $|\alpha|$ denotes the number of elements in $\alpha$ we have

$$
\prod_{j=1}^{r} z_{j}=\sum_{n=0}^{r} \sum_{|\alpha|=n} \prod_{j \in \alpha}\left(z_{j}-1\right)
$$

Put $w_{n}\left(k_{1}, \ldots, k_{n}\right)=\prod_{j=1}^{n}\left(e^{h\left(k_{j}\right)}-1\right)$. It follows from equations (3.2) and (3.3) that if $\psi$ is an $r$ vector in $\mathscr{E}$ then for $n \leqq r$

$$
\begin{aligned}
\left(B_{w_{n}} \psi\right)\left(k_{1}, \ldots, k_{r}\right) & =n !\left(\frac{r !}{(n-r) ! n !}\right) P_{s}\left(w_{n}\left(k_{1}, \ldots, k_{n}\right) \psi\left(k_{1}, \ldots, k_{r}\right)\right) \\
& =n ! \sum_{|\alpha|=n}\left\{\prod_{j \in \alpha}\left(e^{h\left(k_{j}\right)}-1\right)\right\} \psi\left(k_{1}, \ldots, k_{r}\right) .
\end{aligned}
$$

Hence, defining $B_{w_{0}}=I$ and using (3.9) with $z_{j}=e^{h\left(k_{j}\right)}$ we obtain

$$
\left(\sum_{n=0}^{r}(n !)^{-1} B_{w_{n}} \psi\right)\left(k_{1}, \ldots, k_{r}\right)=\prod_{j=1}^{r} e^{h\left(k_{j}\right)} \psi\left(k_{1}, \ldots, k_{r}\right) .
$$


The left side may be summed to $\infty$ instead of to $r$ since the additional terms are zero. The right side is $e^{N(h)} \psi$. Thus for any vector $\psi$ in $\mathscr{E}$ we have

$$
e^{N(h)} \psi=\sum_{n=0}^{\infty}(n !)^{-1} B_{w_{n}} \psi .
$$

Since both sides of this equation contain only operators which are bounded on $r$-particle states the equation clearly holds for these vectors also.

If $\psi$ is the physical rest state and $\psi_{r}$ is its $r$ particle component then $\psi$ is in the domain of $e^{N_{h} / 2}$ for, keeping in mind the non-negativity of $B_{w_{n}}$, as well as the fact that $B_{w_{n}}$ and $e^{N_{h} / 2}$ are the direct sums of their restrictions to the $r$ particle subspaces, we have, in view of (3.5)

$$
\begin{aligned}
\sum_{r=0}^{\infty}\left\|e^{N_{h} / 2} \psi_{r}\right\|^{2} & =\sum_{r=0}^{\infty}\left(e^{N_{h}} \psi_{r}, \psi_{r}\right) \\
& =\sum_{r=0}^{\infty} \sum_{n=0}^{\infty}(n !)^{-1}\left(B_{w_{n}} \psi_{r}, \psi_{r}\right) \\
& =\sum_{n=0}^{\infty}(n !)^{-1} \sum_{r=0}^{\infty}\left(B_{w_{n}} \psi_{r}, \psi_{r}\right) \\
& =\sum_{n=0}^{\infty}(n !)^{-1}\left(B_{w_{n}} \psi, \psi\right) \\
& \leqq \sum_{n=0}^{\infty}(n !)^{-1}\left(\int\left(e^{h(k)}-1\right) f(k)^{2} / \mu(k)^{2} d k\right)^{n} \\
& =c(h)<\infty .
\end{aligned}
$$

(Recall $f$ has compact support.) Replacing $h$ by $2 h$ shows that $\psi$ is in $\mathscr{D}\left(e^{N_{h}}\right)$ and since the left side of the last inequality is $\left(e^{N_{h}} \psi, \psi\right)$ the corollary is proved.

The Sobolev Space $\mathscr{F}^{\prime}(S)$. Let $S$ be an open set in $E_{d}$. Let $N(S)$ denote the number operator on $\mathscr{F}$ associated with $S$. That is,

$$
N(S)=\int_{S} b(k)^{*} b(k) d k .
$$

Let $D=N(S)^{1 / 2}$. $\mathscr{E}$ is clearly a core of $D$ and if $\psi$ is in $\mathscr{D}(D)$ there is a sequence $\psi_{n}$ in $\mathscr{E}$ such that $\psi_{n}$ converges to $\psi$ and $D \psi_{n}$ converges to $D \psi$. Since

$$
\begin{aligned}
\|D \varphi\|^{2} & =(N(S) \varphi, \varphi) \\
& =\int_{S}\|b(k) \varphi\|^{2} d k \quad \varphi \in \mathscr{E}
\end{aligned}
$$

it follows that $b(k) \psi_{n}$, as a function from $S$ to $\mathscr{F}$ converges in $L^{2}(S ; \mathscr{F})$ norm to a function from $S$ to $\mathscr{F}$, which we denote by $b(k) \psi$, and which is well defined up to a set of Lebesgue measure zero in $S$. Since $b(k) \psi_{n}$ 
is a continuous function into $\mathscr{F}, b(k) \psi$ is strongly measurable as a function into $\mathscr{F}$. Of course

$$
\|D \psi\|^{2}=\int_{S}\|b(k) \psi\|^{2} d k
$$

Denote by $C_{c}^{\infty}(S ; \mathscr{F})$ the space of infinitely differentiable functions from $S$ to $\mathscr{F}$ with compact support in $S$. Let $\psi$ be in $\mathscr{D}(D)$. We say that $b(k) \psi$ is weakly differentiable in $L^{2}(S ; \mathscr{F})$ if there are functions $\psi_{j}^{\prime}(k)$ in $L^{2}(S ; \mathscr{F}), j=1, \ldots, d$, such that for all $\varphi$ in $C_{c}^{\infty}(S ; \mathscr{F})$ there holds

$$
-\int_{S}\left(\frac{\partial \varphi}{\partial k_{j}}(k), b(k) \psi\right) d k=\int_{S}\left(\varphi(k), \psi_{j}^{\prime}(k)\right) d k \quad j=1, \ldots, d
$$

where $k_{j}$ are the coordinates of $k$. Such functions $\psi_{j}^{\prime}(k)$ are unique, up to a set of Lebesgue measure zero in $S$, if they exist, since $C_{c}^{\infty}(S ; \mathscr{F})$ is dense in $L^{2}(S ; \mathscr{F})$. We shall write

$$
\frac{\partial}{\partial k_{j}} b(k) \psi=\psi_{j}^{\prime}(k)
$$

if these weak $L^{2}$ derivatives exist.

We denote by $\mathscr{F}^{\prime}(S)$ the set of elements $\psi$ in the domain of $D$ such that $b(k) \psi$ is weakly differentiable in $L^{2}(S ; \mathscr{F})$. For $\psi$ in $\mathscr{F}^{\prime}(S)$ we put

$$
\|\psi\|_{S}^{2}=\|\psi\|^{2}+\|D \psi\|^{2}+\sum_{j=1}^{d} \int\left\|\frac{\partial}{\partial k_{j}} b(k) \psi\right\|^{2} d k .
$$

$\mathscr{F}^{\prime}(S)$ is a Hilbert space in this norm. In order to see that $\mathscr{F}^{\prime}(S)$ is complete note that if $\psi_{n}$ is Cauchy in the $\|\cdot\|_{S}$ norm then $\psi_{n}$ and $D \psi_{n}$ are Cauchy in $\mathscr{F}$. Since $D$ is a closed operator $\psi_{n}$ converges to an element $\psi$ in $\mathscr{D}(D)$ and $D \psi_{n}$ converges to $D \psi$ in $\mathscr{F}$. Furthermore, $\frac{\partial}{\partial k_{j}} b(k) \psi_{n}$ is Cauchy in $L^{2}(S ; \mathscr{F})$ by assumption, and hence has a limit in $L^{2}(S ; \mathscr{F})$ which limit, by (3.11) and (3.12) is $\frac{\partial}{\partial k_{j}} b(k) \psi$. Thus $\psi_{n}$
converges to $\psi$ in \|\|$_{s}$ norm.

Lemma 3.6. Let $k$ and $k^{\prime}$ be in $E_{d}$ and put $\delta b=b\left(k^{\prime}\right)-b(k)$. For any vector $\psi$ in $\mathscr{E}$ there holds

$$
\begin{aligned}
\mu(k)\|(\delta b) \psi\| \leqq 2\left|k-k^{\prime}\right|\left\|b\left(k^{\prime}\right) \psi\right\| & +\left|f(k)-f\left(k^{\prime}\right)\right|\|\psi\| \\
& +\|(\delta b) H(0) \psi\| .
\end{aligned}
$$

Proof. For any vector $\psi$ in $\mathscr{E}$ we have

$$
\begin{aligned}
0 & \leqq(H(-k)(\delta b) \psi,(\delta b) \psi) \\
& =(\{H(-k)(\delta b)-(\delta b) H(0)\} \psi,(\delta b) \psi)+((\delta b) H(0) \psi,(\delta b) \psi) .
\end{aligned}
$$


Since $E$ is even we write its contribution to $H(0)$ as $E(P)$ instead of $E(-P)$. one computes easily that on $\mathscr{E}$

$$
\begin{gathered}
H(-k)(\delta b)-(\delta b) H(0)=E(P+k)(\delta b)-(\delta b) E(P) \\
+\left[\int \mu\left(k^{\prime \prime}\right) b\left(k^{\prime \prime}\right) * b\left(k^{\prime \prime}\right) d k^{\prime \prime}, \delta b\right]+[V, \delta b] \\
=\left(E(P+k)-E\left(P+k^{\prime}\right)\right) b\left(k^{\prime}\right)-\left(\mu\left(k^{\prime}\right) b\left(k^{\prime}\right)-\mu(k) b(k)\right) \\
-\left(f\left(k^{\prime}\right)-f(k)\right) \\
=\left(E(P+k)-E\left(P+k^{\prime}\right)\right) b\left(k^{\prime}\right)-\mu(k) \delta b \\
+\left(\mu(k)-\mu\left(k^{\prime}\right)\right) b\left(k^{\prime}\right)-\left(f\left(k^{\prime}\right)-f(k)\right) .
\end{gathered}
$$

Since $\left|E(p)-E\left(p^{\prime}\right)\right| \leqq\left|p-p^{\prime}\right|$ for all $p$ and $p^{\prime}$ in $E_{d}$ the operator $E(P+k)$ $-E\left(P+k^{\prime}\right)$ is bounded on its domain with norm at most $\left|k-k^{\prime}\right|$. Moreover $\left|\mu(k)-\mu\left(k^{\prime}\right)\right| \leqq\left|k-k^{\prime}\right|$. Combining (3.15) and (3.16) we get

$$
\begin{aligned}
0 \leqq & \left|k-k^{\prime}\right|\left\|b\left(k^{\prime}\right) \psi\right\|\|(\delta b) \psi\|-\mu(k)\|(\delta b) \psi\|^{2} \\
& +\left|k-k^{\prime}\right|\left\|b\left(k^{\prime}\right) \psi\right\|\|(\delta b) \psi\|+\mid f\left(k^{\prime}\right)-f(k)\|\| \psi\|\|(\delta b) \psi \| \\
& +\|(\delta b) H(0) \psi\|\|(\delta b) \psi\| .
\end{aligned}
$$

Upon dividing by $\|(\delta b) \psi\|$ we obtain (3.14).

Lemma 3.7. Let $c$ be a fixed vector in $E_{d}$ and let $\psi$ be the physical rest state with $\|\psi\|=1$. Then for any open set $S$ in $E_{d}$ there holds

$$
\begin{aligned}
& \int_{S} \|(b(k+c)-b(k)) \psi \|^{2} d k \leqq 8|c|^{2} \int_{S} \frac{|f(k+c)|^{2}}{\mu(k)^{2} \mu(k+c)^{2}} d k \\
&+2 \int_{S} \frac{|f(k)-f(k+c)|^{2}}{\mu(k)^{2}} d k .
\end{aligned}
$$

Proof. As shown in the proof of Proposition 3.3 we may pick a sequence $\psi_{j}$ in $\mathscr{E}$ such that $\psi_{j}$ converges to $\psi, b(k) \psi_{j}$ converges to $b(k) \psi$ in $L^{2}\left(E_{d} ; \mathscr{F}\right)$, and $b(k) H(0) \psi$ converges to zero in $L^{2}\left(E_{d} ; \mathscr{F}\right)$. Apply Lemma 3.6 with $k^{\prime}=k+c$ to $\psi_{j}$ yielding

$$
\begin{aligned}
& \left\|(b(k+c)-b(k)) \psi_{j}\right\| \leqq \mu(k)^{-1}\left\{2|c|\left\|b(k+c) \psi_{j}\right\|\right. \\
& \left.\quad+|f(k)-f(k+c)|\left\|\psi_{j}\right\|+\left\|(b(k+c)-b(k)) H(0) \psi_{j}\right\|\right\} .
\end{aligned}
$$

Since $b(k) H(0) \psi_{j}$ and $b(k+c) H(0) \psi_{j}$ converge to zero in $L^{2}\left(E_{d} ; \mathscr{F}\right)$ the last term on the right of (3.18) converges to zero in $L^{2}(S)$. Square both sides of (3.18), integrate over $S$, take the limit as $j \rightarrow \infty$ and then use the inequality $(a+b)^{2} \leqq 2 a^{2}+2 b^{2}$ to obtain

$$
\begin{aligned}
& \int_{S}\|(b(k+c)-b(k)) \psi\|^{2} d k \\
& \quad \leqq 8|c|^{2} \int_{S} \frac{\|b(k+c) \psi\|^{2}}{\mu(k)^{2}} d k+2 \int_{S} \frac{|f(k)-f(k+c)|^{2}}{\mu(k)^{2}} d k .
\end{aligned}
$$


Let $\chi$ denote the characteristic function of $S$. In Proposition 3.3 take $n=1$ and $v(k)=\mu(k-c)^{-2} \chi(k-c)$ to obtain

$$
\int_{E_{d}} \frac{\chi(k-c)\|b(k) \psi\|^{2}}{\mu(k-c)^{2}} d k \leqq \int \frac{\chi(k-c)|f(k)|^{2}}{\mu(k-c)^{2} \mu(k)^{2}} d k .
$$

Translating both integrals in this inequality by $c$ yields an estimate for the first term on the right of (3.19) which establishes the Lemma.

Proposition 3.8. For any open set $S \subset E_{d}$ the physical rest state $\psi$ is in the Sobolev space $\mathscr{F}^{\prime}(S)$. If $\psi$ is normalized to one then

$$
\|\psi\|_{S}^{2} \leqq 1+\int_{S} \frac{|f(k)|^{2}}{\mu(k)^{2}} d k+8 d \int_{S} \frac{|f(k)|^{2}}{\mu(k)^{4}} d k+2 \int_{S} \frac{|\nabla f|^{2}}{\mu(k)^{2}} d k .
$$

Proof. We already know from Proposition 3.3 that $\psi$ is in $\mathscr{D}(N) \subset \mathscr{D}(N(S)) \subset \mathscr{D}(D)$. Let $\varphi \in C_{c}^{\infty}(S ; \mathscr{F})$. Let $e_{j}$ be the $j t h$ basis vector of $E_{d}$. Then

$$
\begin{aligned}
\left|\int_{S}\left(\frac{\partial \varphi(k)}{\partial k_{j}}, b(k) \psi\right) d k\right| \\
=\left|\lim _{s \rightarrow 0} s^{-1} \int_{S}\left(\varphi\left(k+s e_{j}\right)-\varphi(k), b(k) \psi\right) d k\right| \\
=\left|\lim _{s \rightarrow 0} s^{-1} \int_{S}\left(\varphi(k),\left(b\left(k-s e_{j}\right)-b(k)\right) \psi\right) d k\right| \\
\quad \leqq\left(\int_{S}\|\varphi(k)\|^{2} d k\right)^{1 / 2} \lim _{s \rightarrow 0} \sup |s|^{-1}\left(\int_{S}\left\|\left(b\left(k-s e_{j}\right)-b(k)\right) \psi\right\|^{2} d k\right)^{1 / 2} .
\end{aligned}
$$

Put $c=-s e_{j}$ in (3.17). We get

$$
\begin{aligned}
& \lim _{s \rightarrow 0} \sup s^{-2} \int_{S}\left\|\left(b\left(k-s e_{j}\right)-b(k)\right) \psi\right\|^{2} d k \\
& \leqq 8 \int_{S} \frac{|f(k)|^{2}}{\mu(k)^{2}} d k+2 \int_{S} \frac{\left|\frac{\partial f}{\partial k_{j}}(k)\right|^{2}}{\mu(k)^{2}} d k .
\end{aligned}
$$

Thus the left side of (3.21) is a continuous function of $\varphi(\cdot)$ in the norm of $L^{2}(S ; \mathscr{F})$. Hence there is a function $\psi_{j}(\cdot)$ in $L^{2}(S ; \mathscr{F})$ such that $-\int\left(\partial \varphi / \partial k_{j}, b(k) \psi\right) d k=\int\left(\varphi(k), \psi_{j}(k)\right) d k$ for all $\varphi(\cdot)$ in $C_{c}^{\infty}(S ; \mathscr{F})$. Moreover the right side of (3.22) gives an estimate for $\int\left\|\psi_{j}(k)\right\|^{2} d k$. Therefore $\psi$ is weakly differentiable in $S$ and

$$
\sum_{j=1}^{d} \int_{S}\left\|\frac{\partial}{\partial k_{j}} b(k) \psi\right\|^{2} d k \leqq 8 d \int_{S} \frac{|f(k)|^{2}}{\mu(k)^{4}} d k+2 \int_{S} \frac{|\nabla f|^{2}}{\mu(k)^{2}} d k .
$$


Finally the estimate

$$
\int_{S}\|b(k) \psi\|^{2} d k \leqq \int_{S} \frac{|f(k)|^{2}}{\mu(k)^{2}} d k
$$

follows from Proposition 3.3 with $n=1$ and $v$ equal to the characteristic function of $S$.

\section{§ 4. Proofs of Theorems}

Lemma 4.1. Let $r$ be a strictly positive real number and denote by $S$ the cube $S=\left\{k \in E_{d} ;\left|s_{j}\right|<r, j=1, \ldots, d\right\}$ where $s_{j}$ denotes the $j$ th coordinate of $k$. Let $\mathscr{F}_{S}$ denote Fock space over $L^{2}(S)$ and let $\mathscr{F}^{\prime}\left(L^{2}(S)\right)$ denote the set of vectors $\psi$ in $\mathscr{F}_{S}$ which are in the domain of the square root of the number operator $N$ on $\mathscr{F}_{S}$ and such that the weak derivative $\frac{\partial}{\partial s_{j}} b(k) \psi$ exists and is in $L^{2}\left(S ; \mathscr{F}_{S}\right), j=1, \ldots, d$. For such $\psi$ put

$$
\|\psi\|_{1}^{2}=\|\psi\|^{2}+\left\|N^{1 / 2} \psi\right\|^{2}+\sum_{j=1}^{d} \int_{S}\left\|\frac{\partial}{\partial s_{j}} b(k) \psi\right\|^{2} d k .
$$

There exists a positive operator $L$ on $\mathscr{F}_{S}$ with a compact inverse such that $\mathscr{D}(L)=\mathscr{F}^{\prime}\left(L^{2}(S)\right)$ and

$$
\|\psi\|_{1}=\|L \psi\|
$$

for all $\psi$ in $\mathscr{F}^{\prime}\left(L^{2}(S)\right)$.

Proof. We first remark that this lemma is an extension of Rellich's lemma [1] and is valid for any set $S$ with smooth boundary. We have chosen to state it only for a cube since the proof of Rellich's lemma is elementary in that case and we shall include a proof of it here.

We write $\mathscr{F}^{\prime}$ instead of $\mathscr{F}^{\prime}\left(L^{2}(S)\right)$ throughout this proof. One may verify as in the discussion preceding Lemma 3.6 that $\mathscr{F}^{\prime}$ is a Hilbert space in the norm \|\|$_{1}$.

The functions $\cos (n \pi \cdot s /(2 r)), n=0,1,2, \ldots$ form an orthogonal basis of $L^{2}(0,2 r)$ and therefore the functions $u_{n}(s)=c_{n} \cos (n \pi(s+r) /(2 r))$ form an orthonormal basis of $L^{2}(-r, r)$ for suitable constants $c_{n}$. The $u_{n}$ are eigenfunctions of the operator $1-d^{2} / d s^{2}$ with eigenvalues $1+(n \pi / r)^{2}$ and satisfy the boundary conditions $d u_{n} / d s=0$ at $s= \pm r$. If $k=\left(s_{1}, \ldots, s_{d}\right)$ is in the cube $S$ put

$$
w_{n_{1}, \ldots, n_{d}}\left(s_{1}, \ldots, s_{d}\right)=u_{n_{1}}\left(s_{1}\right) \ldots u_{n_{d}}\left(s_{d}\right), n_{j} \geqq 0, \quad j=1, \ldots, d .
$$

The functions $w_{n_{1}, \ldots, n_{d}}$ form an orthonormal basis of $L^{2}(S)$, are eigenfunctions of the operator $1-\Delta$, where $\Delta$ denotes the Laplacian, and 
these functions have normal derivative equal to zero on each face of the cube $S$. We enumerate them $v_{1}, v_{2}, \ldots$ and denote by $\lambda_{j}$ the corresponding eigenvalue: $(1-\Delta) v_{j}=\lambda_{j} v_{j}$. Clearly $\lambda_{j} \rightarrow \infty$ as $j \rightarrow \infty$ and $\lambda_{j} \geqq 1$ for all $j$, since each $\lambda_{j}$ is a sum of $d$ of the eigenvalues given above.

We now construct an orthonormal basis of $\mathscr{F}_{S}$ from the $v_{j}$ in the usual way (cf. [2]). Specifically, for each finite non-decreasing sequence $\alpha$ of positive integers put

$$
\varphi_{\alpha}\left(k_{1}, \ldots, k_{n}\right)=c_{\alpha} P \prod_{j=1}^{n} v_{\alpha(j)}\left(k_{j}\right)
$$

where $n$ is the length of the sequence $\alpha, P$ is the symmetrization projection and $c_{\alpha}$ is a normalization constant chosen to make the $\left\{\varphi_{\alpha}\right\}$ an orthonormal set. If, for the empty sequence $\alpha$, we define $\varphi_{\alpha}$ to be the rank zero tensor 1 then the $\varphi_{\alpha}$ form an orthonormal basis of $\mathscr{F}_{S}$. The functions $\varphi_{\alpha}$ are clearly in $\mathscr{F}^{\prime}$.

Let $A$ be the (unique) self-adjoint operator in $L^{2}(S)$ which is diagonal on the basis $v_{j}$ and multiplies $v_{j}$ by $\lambda_{j}$. Thus $A$ coincides with the operator $1-\Delta$ on the $v_{j}$ (and is in fact the self-adjoint version of the differential operator $1-\Delta$ corresponding to the Neumann boundary condition: normal derivative $=0$ on $\partial S$ ). Let $M$ be the quantization of $A$. That is, $M$ is the closure of the operator which on algebraic $n$ tensors is given by $A \otimes I \otimes \cdots \otimes I+\cdots+I \otimes \cdots \otimes I \otimes A$. One computes easily that the above described basis vector $\varphi_{\alpha}$ is an eigenvector for $M$ with eigenvalue $\mu_{\alpha}=\sum_{j} \lambda_{\alpha(j)}$. Any real number clearly exceeds at most finitely many of the (non-negative) numbers $\mu_{\alpha}$ and therefore $I+M$ has a compact inverse. If $L=(I+M)^{1 / 2}$ then $L$ also has a compact inverse and we shall show that this operator satisfies the requirements of the lemma.

Suppose that $\psi$ is in $\mathscr{F}^{\prime}$. We assert that

$$
\left(\varphi_{\alpha}, \psi\right)_{1}=\left((1+M) \varphi_{\alpha}, \psi\right) .
$$

The principle technical point in this lemma consists in justifying the integration by parts involved in (4.4). Denote by $\partial_{j} S$ that portion of the boundary of $S$ consisting of the two closed faces perpendicular to the $j$ th coordinate axis. Let $g_{m}$ be a sequence of uniformly bounded real valued functions in $C_{c}^{\infty}(S)$ such that $g_{m}(k)=1$ if $\operatorname{dist}(k, \partial S) \geqq m^{-1}$, and such that for some constant $c$ and each $j=1, \ldots, d,\left|\left(\partial / \partial s_{j}\right) g_{m}(k)\right|=0$ if $\operatorname{dist}\left(k, \partial_{j} S\right)$ $>m^{-1}$ while $\left|\left(\partial / \partial s_{j}\right) g_{m}(k)\right| \leqq c m$ if $\operatorname{dist}\left(k, \partial_{j} S\right) \leqq m^{-1}$. The existence of such a sequence is clear if $S$ is one dimensional and a product function will work if $S$ is $d$ dimensional.

$$
\text { Now } \begin{aligned}
\left(\varphi_{\alpha}, \psi\right)_{1}=\left(\varphi_{\alpha}, \psi\right) & +\int_{S}\left(b(k) \varphi_{\alpha}, b(k) \psi\right) d k \\
& +\sum_{j=1}^{d} \int_{S}\left(\frac{\partial}{\partial s_{j}} b(k) \varphi_{\alpha}, \frac{\partial}{\partial s_{j}} b(k) \psi\right) d k
\end{aligned}
$$


where $s_{1}, \ldots, s_{d}$ are the coordinates of $k$. In view of the known form of $\varphi_{\alpha}$ the functions $g_{m}(k) \frac{\partial}{\partial s_{j}} b(k) \varphi_{\alpha}$ are in $C_{c}^{\infty}\left(S ; \mathscr{F}_{S}\right)$. Therefore by the dominated convergence theorem and the definition of $\frac{\partial}{\partial s_{j}} b(k) \psi$ we have

$$
\begin{aligned}
& \int_{S}\left(\frac{\partial}{\partial s_{j}} b(k) \varphi_{\alpha}, \frac{\partial}{\partial s_{j}} b(k) \psi\right) d k \\
&=\lim _{m \rightarrow \infty} \int_{S} g_{m}(k)\left(\frac{\partial}{\partial s_{j}} b(k) \varphi_{\alpha}, \frac{\partial}{\partial s_{j}} b(k) \psi\right) d k \\
&=-\lim _{m \rightarrow \infty} \int_{S}\left(\frac{\partial}{\partial s_{j}}\left(g_{m}(k) \frac{\partial}{\partial s_{j}} b(k) \varphi_{\alpha}\right), b(k) \psi\right) d k \\
&=-\lim _{m \rightarrow \infty} \int_{S} g_{m}(k)\left(\frac{\partial^{2}}{\partial s_{j}^{2}} b(k) \varphi_{\alpha}, b(k) \psi\right) d k \\
&-\lim _{m \rightarrow \infty} \int_{S} \frac{\partial g_{m}(k)}{\partial s_{j}}\left(\frac{\partial}{\partial s_{j}} b(k) \varphi_{\alpha}, b(k) \psi\right) d k \\
&=-\int\left(\left(\frac{\partial^{2}}{\partial s_{j}^{2}} b(k) \varphi_{\alpha}\right), b(k) \psi\right) d k \\
&-\lim _{m \rightarrow \infty} \int_{\operatorname{dist}\left(k, \partial_{j} S\right) \leqq m^{-1}} \frac{\partial g_{m}(k)}{\partial s_{j}}\left(\frac{\partial}{\partial s_{j}} b(k) \varphi_{\alpha}, b(k) \psi\right) d k
\end{aligned}
$$

Now from the form of $\varphi_{\alpha}$ it follows that $\frac{\partial}{\partial s_{j}} b(k) \varphi_{\alpha}$ is zero at all points of $\partial_{j} S$. Moreover all second derivatives of each function $v_{i}$ are bounded on $[-r, r]$ so that $\left\|\frac{\partial}{\partial s_{j}} b(k) \varphi_{\alpha}\right\|$ increases at most linearly with the distance of $k$ from $\partial_{j} S$. In particular there is a constant $c^{\prime}$ such that $\left\|\frac{\partial}{\partial s_{j}} b(k) \varphi_{\alpha}\right\| \leqq c^{\prime} m^{-1}$ if $\operatorname{dist}\left(k, \partial_{j} S\right) \leqq m^{-1}$. Hence the integrand of the last integral is dominated by $\mathrm{cm} \cdot \mathrm{c}^{\prime} \mathrm{m}^{-1}\|b(k) \psi\|$ which is a fixed $L^{2}$ function. Therefore the last limit on $m$ in (4.6) is zero.

It follows then from (4.5) that

$$
\begin{aligned}
\left(\varphi_{\alpha}, \psi\right)_{1} & =\left(\varphi_{\alpha}, \psi\right)+\int_{S}\left((1-\Delta) b(k) \varphi_{\alpha}, b(k) \psi\right) d k \\
& =\left(\varphi_{\alpha}, \psi\right)+n \int_{S}\left(1-\Delta_{1}\right) \varphi_{\alpha}\left(k_{1}, k_{2}, \ldots, k_{n}\right) \psi_{n}\left(k_{1}, k_{2}, \ldots, k_{n}\right) d k_{1} \ldots d k_{n}
\end{aligned}
$$

where $\Delta_{j}$ denotes the Laplacian acting in the $k_{j}$ variable. 
Since $\varphi_{\alpha}$ and $\psi_{n}$ are both symmetric functions $\Delta_{1}$ may be replaced by $\Delta_{j}$ in the last integral yielding

$$
\begin{aligned}
\left(\varphi_{\alpha}, \psi\right)_{1} & =\left(\varphi_{\alpha}, \psi\right)+\int_{S}\left(\sum_{j=1}^{n}\left(1-\Delta_{j}\right) \varphi_{\alpha}\left(k_{1}, \ldots, k_{n}\right)\right) \psi_{n}\left(k_{1}, \ldots, k_{n}\right) d k_{1}, \ldots, d k_{n} \\
& =\left((1+M) \varphi_{\alpha}, \psi\right) .
\end{aligned}
$$

This establishes (4.4).

It now follows from (4.4) that the $\varphi_{\alpha}$ form an orthogonal basis of $\mathscr{F}^{\prime}$. For $\left(\varphi_{\alpha}, \varphi_{\beta}\right)_{1}=\left((1+M) \varphi_{\alpha}, \varphi_{\beta}\right)=\left(1+\mu_{\alpha}\right)\left(\varphi_{\alpha}, \varphi_{\beta}\right)=\left(1+\mu_{\alpha}\right) \delta_{\alpha, \beta}$ and if $\psi$ is in $\mathscr{F}^{\prime}$ and $\left(\varphi_{\alpha}, \psi\right)_{1}=0$ for all $\alpha$ then $\left(1+\mu_{\alpha}\right)\left(\varphi_{\alpha}, \psi\right)=0$ for all $\alpha$ and therefore $\psi=0$. Hence the vectors $\left(1+\mu_{\alpha}\right)^{-1 / 2} \varphi_{\alpha}$ form an orthonormal basis of $\mathscr{F}^{\prime}$. Thus $\psi$ is in $\mathscr{F}^{\prime}$ if and only if $\psi$ has the form

$$
\psi=\sum_{\alpha} c_{\alpha}\left(1+\mu_{\alpha}\right)^{-1 / 2} \varphi_{\alpha}
$$

where $\Sigma\left|c_{\alpha}\right|^{2}<\infty$ and the convergence is in $\mathscr{F}^{\prime}$ and therefore also in $\mathscr{F}$ norm. Since $L$ is a closed operator which is diagonal on $\left\{\varphi_{\alpha}\right\}$ and $L \varphi_{\alpha}=\left(1+\mu_{\alpha}\right)^{1 / 2} \varphi_{\alpha}$ it follows from (4.7) that the domain of $L$ is exactly $\mathscr{F}^{\prime}$ and for the above vector $\psi\|L \psi\|^{2}=\Sigma\left|c_{\alpha}\right|^{2}=\|\psi\|_{1}^{2}$.

The following lemma is a slight, but essential, modification of a lemma used in [6].

Lemma 4.2. Let $\mathscr{T}$ be a family of non-negative trace class operators on a Hilbert space $X$ and let $L$ be a positive self-adjoint operator on $X$ with compact inverse such that

1. $L T L$ is densely defined and bounded for all $T$ in $\mathscr{T}$ and its closure $(L T L)^{-}$is trace class.

2. There is a real number a such that trace $\left((L T L)^{-}\right)<$a for all $T$ in $\mathscr{T}$.

Then $\mathscr{T}$ is relatively compact in trace class norm.

Proof. We show that $\mathscr{T}$ is totally bounded in trace class norm. Let $\varepsilon>0$. Let $b=\left\|L^{-1}\right\|$. Choose $c>0$ such that $\max \left(b^{-1} c^{-1} a, c^{-2} a\right)$ $<\varepsilon / 12$. Let $E_{1}$ be the spectral projection of $L$ for the interval $[0, c]$. Let $E_{2}=1-E_{1}$. Let $T$ be in $\mathscr{T}$ and let $A$ be the closure of $L T L$. Then $T=L^{-1} A L^{-1}$ and $E_{i} T E_{j}=L^{-1} E_{i} A L^{-1} E_{j}$ for all $i, j$. Denoting the trace class norm by \|\|$_{t}$ we have $\left\|E_{i} T E_{j}\right\|_{t} \leqq\left\|L^{-1} E_{i}\right\|\|A\|_{t}\left\|L^{-1} E_{j}\right\|$. Since $\left\|L^{-1} E_{2}\right\| \leqq c^{-1},\left\|L^{-1} E_{1}\right\| \leqq b^{-1}$, and $\|A\|_{t} \leqq a$, it follows that $\left\|E_{i} T E_{j}\right\|_{t}<\varepsilon / 12$ if $i$ or $j$ is 2 and $\left\|E_{1} T E_{1}\right\|_{t} \leqq b^{-2} a$. Since $L^{-1}$ is compact $E_{1}$ is finite dimensional. Hence the set $\mathscr{S}=\left\{E_{1} T E_{1}: T \in \mathscr{T}\right\}$ is relatively compact in trace class norm. Thus there exists a finite set of operators $T_{1}, \ldots, T_{n}$ in $\mathscr{T}$ such that $\left\{E_{1} T_{k} E_{1}\right\}_{k=1}^{n}$ is an $\varepsilon / 2$ net for $\mathscr{S}$. If $T$ is any 
operator in $\mathscr{T}$ then

$$
\begin{aligned}
\left\|T-T_{k}\right\|_{t} & \leqq \sum_{i, j=1}^{2}\left\|E_{i}\left(T-T_{k}\right) E_{j}\right\|_{t} \\
& \leqq\left\|E_{1}\left(T-T_{k}\right) E_{1}\right\|_{t}+\sum_{(i, j) \neq(1,1)}\left(\left\|E_{i} T E_{j}\right\|_{t}+\left\|E_{i} T_{k} E_{j}\right\|_{t}\right) \\
& <\left\|E_{1}\left(T-T_{k}\right) E_{1}\right\|_{t}+3 \cdot 2 \cdot \varepsilon / 12 .
\end{aligned}
$$

Hence for some $k\left\|T-T_{k}\right\|_{t}<\varepsilon$. Thus $\mathscr{T}$ is totally bounded.

Proof of Theorem 1. If $U$ is a bounded open set in $E_{d}$ and $S$ is a cube containing $U$ then $\mathscr{A}(U) \subset \mathscr{A}(S)$. Since the norm of a linear functional on $\mathscr{A}(S)$ does not increase when restricted to the subspace $\mathscr{A}(U)$ it suffices to show that the set of restrictions $\omega_{n} \mid \mathscr{A}(S)$ is relatively compact in norm when $S$ is a cube of the type described in Lemma 4.1. We choose a fixed cube $S$ henceforth.

Denote by $S^{c}$ the complement of $S$ in $E_{d}$ and by $\mathscr{F}_{S^{c}}$ the Fock space over $L^{2}\left(S^{c}\right)$. As is known, there is a Hilbert space isomorphism between the Fock space $\mathscr{F}$ over $L^{2}\left(E_{d}\right)$ and the tensor product $\mathscr{F}_{S} \otimes \mathscr{F}_{S^{c}}$ which preserves the form of annihilation operators. In this decomposition of $\mathscr{F}$ the algebra $\mathscr{A}(S)$ is exactly $\mathscr{B}\left(\mathscr{F}_{S}\right) \otimes I$ where $\mathscr{B}\left(\mathscr{F}_{S}\right)$ is the algebra of all bounded operators on $\mathscr{F}_{S}$.

Now if $X$ and $Y$ are Hilbert spaces and $\psi$ is a unit vector in $X \otimes Y$, then, to the state $\omega$ on $\mathscr{B}(X)$ given by $\omega(B)=(B \otimes I \psi, \psi)$ for $B$ in $\mathscr{B}(X)$, there corresponds a unique density matrix $T$ on $X$, i.e., a non-negative trace class operator with trace equal to one, such that

$$
\omega(B)=\operatorname{trace}(T B) \quad B \in \mathscr{B}(X) \text {. }
$$

For if $\psi$ is any vector in $X \otimes Y$ then $\psi$ may be written $\psi=\sum_{n} x_{n} \otimes y_{n}$ where the $y_{n}$ are orthonormal and $\Sigma\left\|x_{n}\right\|^{2}<\infty$, so that $((B \otimes I) \psi, \psi)$ $=\sum_{n}\left(B x_{n}, x_{n}\right)=\operatorname{trace}(T B)$ where $T=\sum_{n}\left\|x_{n}\right\|^{2} P_{n}$ and $P_{n}$ is the onedimensional projection onto the span of $x_{n}$. Clearly trace $T=\sum_{n}\left\|x_{n}\right\|^{2}$ $=\|\psi\|^{2}$. Moreover if $L$ is a self-adjoint operator on $X$ with bounded inverse and if $\psi$ is in the domain of the self-adjoint operator $L \otimes I$ then $L T L$ is densely defined and bounded and its closure is the trace class operator on $X$ corresponding as above to the vector $(L \otimes I) \psi$. For if we define $A$ to be the trace class operator on $X$ corresponding to $(L \otimes I) \psi$ then for any $B$ in $\mathscr{B}(X)$ we have

$$
\begin{aligned}
\operatorname{trace}\left(B L^{-1} A L^{-1}\right) & =\operatorname{trace}\left(L^{-1} B L^{-1} A\right) \\
& =\left(\left(L^{-1} B L^{-1} \otimes I\right)(L \otimes I) \psi,(L \otimes I) \psi\right) \\
& =((B \otimes I) \psi, \psi) \\
& =\operatorname{trace}(B T) .
\end{aligned}
$$


Since $\mathscr{B}(X)$ is the dual space of the Banach space of trace class operators in the pairing $\langle B, T\rangle=\operatorname{trace}(B T)$ it follows that $T=L^{-1} A L^{-1}$ and therefore $L T L$ is densely defined with closure $A$.

To prove the theorem it suffices to show that the sequence $T_{n}$ of density matrices on $\mathscr{F}_{S}$ corresponding to $\psi_{n}$ as in the preceding discussion is compact in trace class norm. In view of Lemma 4.2 and the preceding discussion it suffices to prove that there is a positive self-adjoint operator $L$ on $\mathscr{F}_{S}$ with compact inverse and a real number a such that $\psi_{n}$ is in $\mathscr{D}(L \otimes I)$ for all $n$ and $\left\|(L \otimes I) \psi_{n}\right\| \leqq a$ for all $n$. Choose $L$ as in Lemma 4.1. In view of Lemma 4.1, Eqs. (4.1), (4.2), Proposition 3.8, Eqs. (3.13) and (3.20) it follows that

$$
\begin{aligned}
\left\|(L \otimes I) \psi_{n}\right\|^{2} \leqq & +\int_{S} \frac{\left|f_{n}(k)\right|^{2}}{\mu(k)^{2}} d k+8 d \int_{S} \frac{\left|f_{n}(k)\right|^{2}}{\mu(k)^{4}} d k \\
& +2 \int_{S} \frac{\left|\nabla f_{n}\right|^{2}}{\mu(k)^{2}} d k .
\end{aligned}
$$

Since $S$ is bounded and each of the integrals on the right of (4.8) is, by assumption, uniformly bounded in $n$ on $S$ the theorem is proved.

Proof of Corollary 1.1. The proof is a slight variant of arguments used in [6]. Choose an increasing sequence $S_{m}$ of bounded open sets in $E_{d}$ whose union is $E_{d}$. For any bounded open set $S \subset E_{d}, \mathscr{A}(S) \subset \mathscr{A}\left(S_{m}\right)$ for some $m$. For each $m$ the norm closure $A_{m}$ of the set of restrictions $\left\{\omega_{n} \mid \mathscr{A}\left(S_{m}\right)\right\}_{n=1}^{\infty}$ is norm compact by Theorem 1 , therefore weakly compact and therefore weakly closed. Hence $\omega \mid \mathscr{A}\left(S_{m}\right)$ lies in $A_{m}$ and is a norm limit of some subsequence. By diagonalization there is a subsequence $\omega_{n_{j}}$ such that for every $m \omega_{n_{j}} \mid \mathscr{A}\left(S_{m}\right)$ converges in norm to $\omega \mid \mathscr{A}\left(S_{m}\right)$. Then $\omega_{n_{j}} \mid \mathscr{A}(S)$ converges in norm to $\omega \mid \mathscr{A}(S)$ for any bounded open set $S$ in $E_{d}$ and since $\omega_{n_{j}}(A)$ converges to $\omega(A)$ for each operator $A$ in a norm dense subset of $\mathscr{A}$ it converges to $\omega(A)$ for all $A$ in $\mathscr{A}$.

Factoring $\mathscr{F}=\mathscr{F}_{S} \otimes \mathscr{F}_{S^{c}}$ as in the proof of Theorem 1, and putting $T_{j}$ for the trace class operator on $\mathscr{F}_{S}$ corresponding to $\omega_{n_{j}} \mid \mathscr{A}(S)$ we note that Theorem 1 implies that $T_{j}$ converges in trace class norm to a density matrix $T$ on $\mathscr{F}_{S}$ corresponding to $\omega \mid \mathscr{A}(S)$. Of course $\omega \mid \mathscr{A}(S)$ is also given by a (non-unique) density matrix $T_{S}$ on $\mathscr{F}$ which may be constructed from $T$ and any unit vector $u$ in $\mathscr{F}_{S^{c}}$ via the prescription $T_{S} x=\sum_{n}\left(x, x_{n} \otimes u\right) y_{n} \otimes u$ for $x$ in $\mathscr{T}$, where $T z=\sum_{n}\left(z, x_{n}\right) y_{n}$ for $z$ in $\mathscr{F}_{S}$ and $\Sigma\left\|x_{n}\right\|^{2}<\infty$ and $\Sigma\left\|y_{n}\right\|^{2}<\infty$. Clearly $\omega(A)=\operatorname{trace}\left(A T_{S}\right)$ for $A$ in $\mathscr{A}(S)$ and hence $\omega \mid \mathscr{A}(S)$ is ultraweakly continuous. Thus if $S \subset S_{m}$ and $C$ and $D$ are in $\mathscr{A}\left(S_{m}\right)$ then the map $A \rightarrow\left(\sigma(A) \sigma(C) \psi_{0}, \sigma(D) \psi_{0}\right)$ is ultraweakly continuous on the unit ball of $\mathscr{A}(S)$ since $\left(\sigma(A) \sigma(C) \psi_{0}, \sigma(D) \psi_{0}\right)$ $=\omega\left(D^{*} A C\right)=\operatorname{trace}\left(C T_{S_{m}} D^{*} A\right)$. Since $\left\{\sigma(C) \psi_{0} ; C \in \cup_{m} \mathscr{A}\left(S_{m}\right)\right\}$ is dense 
in $\mathscr{K}_{0},(\omega(A) v, w)$ is ultraweakly continuous for all $v$ and $w$ in $\mathscr{K}_{0}$ on the unit ball of $\mathscr{A}(S)$ and therefore, by [4, Theorem 1 (ii), p. 40], $(\sigma(A) v, w)$ is ultraweakly continuous on all of $\mathscr{A}(S)$. Since $\mathscr{A}\left(S_{m}\right)$ has a countable dense set in the ultraweak topology (e.g. operators corresponding to matrices with finitely non-zero complex rational entries relative to some fixed O.N. basis of $\mathscr{F}_{S_{m}}$, and the map $A \rightarrow\left(\sigma(A) \psi_{0}, w\right)$ is ultraweakly continuous on $\mathscr{A}\left(S_{m}\right)$ for any vector $w$ in $\mathscr{K}_{0}, \mathscr{K}_{0}$ is separable. Since the type I factor $\mathscr{A}(S)$ contains no ultraweakly closed proper two sided ideals, $\sigma \mid \mathscr{A}(S)$ is an isomorphism. Moreover since the unit ball of $\mathscr{A}(S)$ is ultraweakly compact its image under $\sigma$, which we have shown to be ultraweakly-weakly continuous, is weakly compact and therefore weakly closed. Hence $\sigma(\mathscr{A}(S))$ is weakly closed by [4, Theorem 2, p. 43]. Thus $\sigma(\mathscr{A}(S))$ is also a factor of type I. Now $\mathscr{A}(S)$ has countably infinite multiplicity on $\mathscr{F}$. If $S_{m}$ contains $S$ properly then $\mathscr{A}(S)$ has infinite multiplicity in $\mathscr{A}\left(S_{m}\right)$. Hence $\sigma(\mathscr{A}(S))$ has infinite multiplicity in $\sigma\left(\mathscr{A}\left(S_{m}\right)\right)$. Thus $\sigma(\mathscr{A}(S))$ has (necessarily countable) infinite multiplicity on $\mathscr{K}_{0}$. Therefore there exists a unitary operator $U_{S}$ from $\mathscr{F}$ to $\mathscr{K}_{0}$ which implements $\sigma \mid \mathscr{A}(S)$.

Proof of Corollary 1.2. For any bounded open set $B$ in $E_{d}$ let $U_{B}$ be a unitary operator from $\mathscr{F}$ to $\mathscr{K}_{0}$ implementing $\sigma \mid \mathscr{A}(B)$ as in Corollary 1.1. Define $\hat{\sigma}$ on $\mathscr{C}(B)$ by $\hat{\sigma}(D)=\left(I \otimes_{m} U_{B}\right) D\left(I \otimes_{m} U_{B}\right)^{-1}$. Then clearly (2.9) holds for all $\mathscr{C}$ in $\mathscr{B}\left(L^{2}\left(R_{d}\right)\right)$ and $A$ in $\mathscr{A}(B)$. $\hat{\sigma}$ is strongly continuous on the unit ball of $\mathscr{C}(B)$ to the weak operator topology. Since the algebra generated by $\left\{C \otimes A: C \in \mathscr{B}\left(L^{2}\left(R_{d}\right)\right), A \in \mathscr{A}(B)\right\}$ is ultrastrongly dense in $\mathscr{C}(B)$ and any strongly continuous linear functional on the unit ball of $\mathscr{C}(B)$ is ultrastrongly continuous on $\mathscr{C}(B)[4$, p. 38] $\hat{\sigma} \mid \mathscr{C}(B)$ is uniquely determined by conditions a) and b) of the corollary. Thus $\hat{\sigma}$ is independent of the choice of $U_{B}$. Consequently $\hat{\sigma}$ is now a well defined isometric isomorphism on $\cup_{B} \mathscr{C}(B)$ and extends uniquely to an isomorphism on $\mathscr{C}$.

Proof of Proposition 2.2. We write $\mathscr{F}=\mathscr{F}_{B} \otimes \mathscr{F}_{B^{c}}$ as in the proof of Theorem 1. In the decomposition $\mathscr{K}=\left(L^{2}\left(R_{d}\right) \otimes \mathscr{F}_{B}\right) \otimes \mathscr{F}_{B^{c}}$ the algebra $\mathscr{C}(B)$ is exactly $\mathscr{B}\left(L^{2}\left(E_{d}\right) \otimes \mathscr{F}_{B}\right) \otimes I$. To show that a bounded operator $C$ is in $\mathscr{C}(B)$ it suffices to prove that $C$ commutes with all bounded operators of the form $I \otimes I \otimes A$ where $A$ runs over the bounded operators on $\mathscr{F}_{B^{c}}$ or at least over an irreducible set. Let $g$ be a complex function in $L^{2}\left(E_{d}\right)$. For each vector $x$ in configuration space $E_{d}^{\prime}$ denote by $S_{x}(g)$ the selfadjoint operator on $\mathscr{F}$ given by

$$
S_{x}(g)=\int_{E_{d}}\left(e^{i x \cdot k} \overline{g(k)} b(k)+e^{-i x \cdot k} g(k) b(k)^{*}\right) d k .
$$

Relative to the total momentum decomposition (2.3) put

$$
T(g)=I \otimes_{m} S_{0}(g) .
$$


Then in view of the known irreducibility properties [2, Theorem 9] of the creation and annihilation operators it suffices to prove that $C$ commutes with all operators of the form $e^{i T(g)}$ where $g$ has support in $B^{c}$.

In order to show that $e^{i R\left(g^{\prime}\right)}$ and $e^{i T(g)}$ commute when $g$ and $g^{\prime}$ have disjoint supports it will be necessary to make explicit the relation between the two decompositions (1.5) and (2.3) of $\mathscr{K}$ in terms of which the operators $R\left(g^{\prime}\right)$ and $T(g)$ have been defined. First of all, via a Fourier transformation on the first factor in (1.5) we may identify $\mathscr{K}$ with $L^{2}\left(E_{d}^{\prime}\right) \otimes \mathscr{F}$ or, equivalently, with functions $\psi(x)$ on $E_{d}^{\prime}$ with values in $\mathscr{F}$, where $E_{d}^{\prime}$ is configuration space (i.e. the dual space of $E_{d}$ ). $\mathscr{E}_{1}$ is carried into a similarly defined submanifold $\mathscr{E}_{1}^{\prime}$ of $L^{2}\left(E_{d}^{\prime}\right) \otimes \mathscr{F}$. We regard $a(k)$ as defined on $\mathscr{E}_{1}^{\prime}$.

In order to make the argument clearer we first outline the rest of the proof in an informal style ignoring all domain questions. Denote by $I \otimes_{m} b(k)$ the operator on $\mathscr{K}$ defined with respect to the decomposition (2.3). The subscript $m$ refers to the total momentum decomposition (2.3) of $\mathscr{K}$ as noted before. Identifying $L^{2}\left(E_{d}^{\prime}\right) \otimes \mathscr{F}$ with functions $\psi(\cdot)$ from $E_{d}^{\prime}$ to $\mathscr{F} a(k)$ is given by

$$
(a(k) \psi)(x)=b(k)(\psi(x)) .
$$

The identification map $W$ from the momentum decomposition $L^{2}\left(R_{d}\right) \otimes \mathscr{F} \equiv L^{2}\left(R_{d} ; \mathscr{F}\right)$ to $\mathscr{K}=L^{2}\left(E_{d}^{\prime} ; \mathscr{F}\right)$ is given by $W \chi=\psi$ where

$$
\psi(x)=(2 \pi)^{-d / 2} e^{i x \cdot P} \int_{R_{d}} e^{-i p \cdot x} \chi(p) d p
$$

and $\chi$ is a function from $R_{d}$ to $\mathscr{F}$. By definition $\left(\left(I \otimes_{m} b(k)\right) \chi\right)(p)=b(k)(\chi(p))$. Consequently, since

$$
e^{i x \cdot P} b(k)=e^{i x \cdot k} b(k) e^{i x \cdot P}
$$

we have

$$
\begin{aligned}
\left(\left(I \otimes_{m} b(k)\right) \psi\right)(x) & =(2 \pi)^{-d / 2} e^{i x \cdot P} \int e^{-i x \cdot p} b(k) \chi(p) d p \\
& =e^{i x \cdot k} b(k)(\psi(x)) .
\end{aligned}
$$

Comparing this with (4.10) we see that if $k$ is in $B$ and $k^{\prime}$ is in $B^{c}$ then $a\left(k^{\prime}\right)$ commutes with both $I \otimes_{m} b(k)$ and its adjoint:

$$
\left(\left(I \otimes_{m} b(k)\right)^{*} \psi\right)(x)=e^{-i x \cdot k} b(k)^{*}(\psi(x)) .
$$

This informally proves the first assertion of the proposition.

We shall now repeat the preceding argument in a formal style. Since $R\left(g^{\prime}\right)$ acts only on the second factor $e^{i R\left(g^{\prime}\right)}$ operates only on the values of $\psi(x)$ :

$$
\left(e^{i R\left(g^{\prime}\right)} \psi\right)(x)=e^{i S_{0}\left(g^{\prime}\right)}(\psi(x)) \text { for each } x \text { in } E_{d}^{\prime} .
$$


Denote by $F$ Fourier transform regarded as a unitary operator from $L^{2}\left(R_{d}\right)$ to $L^{2}\left(E_{d}^{\prime}\right)$. Let $G$ denote the operator on $L^{2}\left(E_{d}^{\prime}\right) \otimes \mathscr{F}$ to itself given by $(G \psi)(x)=e^{i x \cdot P}(\psi(x))$ where $P$ is the total Boson momentum operator. Then the unitary operator

$$
W=G(F \otimes I)
$$

from $L^{2}\left(R_{d}\right) \otimes \mathscr{F}$ onto $L^{2}\left(E_{d}^{\prime}\right) \otimes \mathscr{F}$ gives the total momentum decomposition of $\mathscr{K}$ i.e., is the map by means of which we identify $L^{2}\left(R_{d}\right) \otimes \mathscr{F}$ with $\mathscr{K}\left[8\right.$, Proposition 6]. Thus, as an operator on $L^{2}\left(E_{d}^{\prime}\right) \otimes \mathscr{F}$ the operator $e^{i T(g)}$ is given by $W e^{i I \otimes S_{0}(g)} W^{-1}=G(F \otimes I) e^{i I \otimes S_{0}(g)}\left(F^{-1} \otimes I\right) G^{-1}$ $=G\left(I \otimes e^{i S_{0}(g)}\right) G^{-1}$. All three of these factors act only on the values of $\psi(x)$. Thus

$$
\begin{aligned}
\left(W e^{i T(g)} W^{-1} \psi\right)(x) & =e^{i x \cdot P} e^{i S_{0}(g)} e^{-i x \cdot P} \psi(x) \\
& =e^{i e^{i x \cdot P} S_{0}(g) e^{-i x \cdot P}} \psi(x) \\
& =e^{i S_{x}(g)} \psi(x)
\end{aligned}
$$

The last equation follows from the Eq. (4.12), which is valid on $\mathscr{E}$. Since $e^{i S_{0}\left(g^{\prime}\right)}$ commutes with $e^{i S_{x}(g)}$ for every $x$ when $g^{\prime}$ is supported in $B$ and $g$ is supported in $B^{c}$ the first assertion of the proposition now follows from (4.13) and (4.14).

In order to prove the second assertion of the proposition let $h$ be a bounded real valued measurable function with support in $B$. The selfadjoint operator

$$
B_{h}=\int_{E_{d}} h(k) b(k)^{*} b(k) d k
$$

on $\mathscr{F}$ has the property that $e^{i t B_{h}}$ lies in $\mathscr{A}(B)$ and so $e^{i t\left(I \otimes_{m} B_{h}\right)}=I \otimes_{m} e^{i t B_{h}}$ lies in $\mathscr{C}(B)$. Denote by $\mathscr{E}_{1}^{\prime \prime}$ the set of functions $\chi(\cdot)$ from $R_{d}$ to $\mathscr{F}$ such that $\chi_{n}\left(p, k_{1}, \ldots, k_{n}\right)$ is zero for large $n$ and in $\mathscr{S}\left(R_{d} \times\left(E_{d}\right)^{n}\right)$ for all $n$. One sees readily that $W$ takes $\mathscr{E}_{1}^{\prime \prime}$ onto $\mathscr{E}_{1}^{\prime}$. For $\varphi$ and $\chi$ in $\mathscr{E}_{1}^{\prime \prime}$ one sees from (4.10), (4.11) and (4.12) and from the unitarity of $e^{i x \cdot P}$ and unitarity of the Fourier transform that

$$
\begin{aligned}
\left(\int h(k) a(k)^{*} a(k) d k W \chi, W \varphi\right) & =\int h(k)(a(k) W \chi, a(k) W \varphi) d k \\
& =\int_{E_{d}} h(k) \int_{\boldsymbol{R}_{d}}(b(k) \chi(p), b(k) \varphi(p)) d p d k \\
& =\left(\left(I \otimes_{m} B_{h}\right) W \chi, W \varphi\right)
\end{aligned}
$$

for any bounded real valued measurable function $h$ on $E_{d}$. Since $\mathscr{E}_{1}^{\prime}$ is a core for $\int h(k) a(k)^{*} a(k) d k$ we have

$$
D_{h}=\int_{E_{d}} h(k) a(k)^{*} a(k) d k=I \otimes_{m} B_{h} .
$$

Hence $e^{i t D_{h}}$ lies in $\mathscr{C}(B)$ when $h$ is supported in $B$. 
Proof of Theorem 2. We first prove part a). If $g$ is a complex valued square integrable function with compact support in $E_{d}$ then by Proposition 2.2 and Corollary 1.2 (b) $\hat{\sigma}\left(e^{i t R(g)}\right)$ is a weakly continuous function of $t$. Hence by Stone's theorem there exists a unique self-adjoint operator $S(g)$ on $\mathscr{K}_{p h}$ such that ii) holds. Since $\hat{\sigma}$ is an isomorphism the operators $S(g)$ satisfy the same commutation relations that the $R(g)$ do - namely (2.15), when $g$ and $g^{\prime}$ have compact support.

Now let $g$ be an arbitrary function in $K_{\tau}$. Let $g_{n}(k)=g(k)$ if $|k| \leqq n$ and zero otherwise. Then $S\left(g_{n}\right)$ is well defined by the preceding paragraph and we shall show that the unitary groups $e^{i t S\left(g_{n}\right)}$ converge strongly on $\mathscr{K}_{p h}$ and uniformly with respect to $t$ on bounded $t$ sets. To this end it suffices to prove that for any bounded open set $B$ in $E_{d}$ and any operator $A$ in $\mathscr{A}(B)$ and any strictly positive number $\varepsilon$ and any vector $u$ in $L^{2}\left(E_{d}\right)$ and any positive real number $T$ there is an integer $n_{1}$ such that

$$
\left\|\left(e^{i t S\left(g_{n}\right)}-e^{i t S\left(g_{m}\right)}\right) u \otimes \sigma(A) \psi_{0}\right\|<\varepsilon
$$

whenever $n$ and $m$ are greater than $n_{1}$ and $|t| \leqq T$. For finite linear combinations of vectors of the form $u \otimes \sigma(A) \psi_{0}$ (with $B$ varying) are dense in $\mathscr{K}_{p h}$ so that the validity of (4.16) would show that the unitary groups $e^{i t S\left(g_{n}\right)}$ converge on a dense set uniformly on bounded $t$ sets and consequently converge everywhere on $\mathscr{K}_{p h}$ uniformly on bounded $t$ sets. The limit will therefore be a strongly continuous unitary group. We may and shall define $S(g)$ as the infinitesimal generator of this group.

Suppose $n \geqq m$. Then $\left(g_{n}, g_{m}\right)=\left\|g_{m}\right\|^{2}$ which is real. Hence by (2.15)

$$
\begin{aligned}
& \left\|\left(e^{i t S\left(g_{n}\right)}-e^{i t S\left(g_{m}\right)}\right) u \otimes \sigma(A) \psi_{0}\right\| \\
= & \left\|\left(e^{i t S\left(g_{n}-g_{m}\right)}-I\right) u \otimes \sigma(A) \psi_{0}\right\| .
\end{aligned}
$$

If $n \geqq m>\sup \{|k| ; k \in B\}$ then the support of $g_{n}-g_{m}$ is disjoint from $B$ and by the proof of Proposition $2.2 e^{i t R\left(g_{n}-g_{m}\right)}$ commutes with $I \otimes_{m} A$, this product being defined relative to the decomposition (2.3). Hence $e^{i t S\left(g_{n}-g_{m}\right)}$ commutes with $I \otimes \sigma(A)$ and we have

$$
\begin{aligned}
& \left\|\left(e^{i t S\left(g_{n}\right)}-e^{i t S\left(g_{m}\right)}\right) u \otimes \sigma(A) \psi_{0}\right\| \\
\leqq & \|A\|\left\|\left(e^{i t S\left(g_{n}-g_{m}\right)}-I\right) u \otimes \psi_{0}\right\|
\end{aligned}
$$

for all sufficiently large $n$ and $m$.

In order to estimate the right side of (4.18) we first show that if $B_{1}$ is any bounded open set in $E_{d}$ and $D$ is in $\mathscr{C}\left(B_{1}\right)$ then

$$
\left(\hat{\sigma}(D) u \otimes \psi_{0}, u \otimes \psi_{0}\right)=\lim _{j \rightarrow \infty}\left(D u \otimes \psi_{n_{j}}, u \otimes \psi_{n_{j}}\right)
$$

where $\psi_{n_{j}}$ is the sequence of physical rest states whose $C^{*}$ algebra limit is the state $\omega$ of $\mathscr{A}$ with respect to which $\mathscr{K}_{0}$ and $\mathscr{K}_{p h}$ have been defined. 
Eq. (4.19) clearly holds if $D$ is of the form $D=C \otimes A$ where $C$ is in $\mathscr{B}\left(L^{2}\left(R_{d}\right)\right)$ and $A$ is in $\mathscr{A}\left(B_{1}\right)$ by Eq. (2.9). Consequently it holds also for finite sums of such products. Such finite sums are strongly dense in $\mathscr{C}\left(B_{1}\right)$ while the left side of (4.19) is strongly continuous on bounded sets in $\mathscr{C}\left(B_{1}\right)$ by Corollary $(1.2 \mathrm{~b})$. Thus if $D$ is in $\mathscr{C}\left(B_{1}\right)$ there is a bounded net $D_{\alpha}$ of finite sums of products, by the Kaplansky density theorem, such that $D_{\alpha} \rightarrow D$ strongly. Thus

$$
\left(\hat{\sigma}(D) u \otimes \psi_{0}, u \otimes \psi_{0}\right)=\lim _{\alpha} \lim _{j}\left(D_{\alpha} u \otimes \psi_{n_{j}}, u \otimes \psi_{n_{j}}\right) .
$$

Since the states of $\mathscr{A}\left(B_{1}\right)$ determined by the vectors $\psi_{n_{j}}$ converge in trace class norm, the (unnormalized) states of $\mathscr{C}\left(B_{1}\right)$ determined by the vectors $u \otimes \psi_{n_{j}}$ also converge in trace class norm. Thus, since the operators $D_{\alpha}$ are bounded, the two limits on the right of (4.20) may be interchanged, yielding (4.19).

Replacing $D$ by $D^{*} D$ in (4.19) we get $\left\|\hat{\sigma}(D) u \otimes \psi_{0}\right\|=\lim _{j}\left\|D u \otimes \psi_{n_{j}}\right\|$ and consequently, for any function $h$ in $K_{\tau}$ with compact support we have

$$
\left\|\left(e^{i t S(h)}-I\right) u \otimes \psi_{0}\right\|=\lim _{j}\left\|\left(e^{i t R(h)}-I\right) u \otimes \psi_{n_{j}}\right\| .
$$

Let

$$
Z=\int_{E_{d}} \mu(k)^{-\tau} a(k)^{*} a(k) d k .
$$

Then in view of Eq. (4.15) and Proposition $3.3 u \otimes \psi_{n}$ is in the domain of $Z$ for each physical rest state $\psi_{n}$ and arbitrary $u$ in $L^{2}\left(E_{d}\right)$. Hence

$$
\begin{aligned}
\left\|\left(e^{i t R(h)}-I\right) u \otimes \psi_{n}\right\| & \\
& \leqq\left\|\left(e^{i t R(h)}-I\right)(Z+1)^{-1 / 2}\right\|\left\|(Z+1)^{1 / 2} u \otimes \psi_{n}\right\| .
\end{aligned}
$$

We now estimate the first factor on the right of the last inequality. Since the domain of $R(h)$ contains the range of $(Z+1)^{-1 / 2}$ we have

$$
\begin{aligned}
\left\|\left(e^{i t R(h)}-I\right)(Z+1)^{-1 / 2} \psi\right\| & =\left\|\int_{0}^{t} e^{i S R(h)} i R(h)(Z+1)^{-1 / 2} \psi d s\right\| \\
& \leqq T\left\|R(h)(Z+1)^{-1 / 2}\right\|\|\psi\|
\end{aligned}
$$

whenever $|t| \leqq T$ and for all $\psi$ in $\mathscr{K}$.

We assert that for any $g$ in $K_{\tau}$

$$
\left\|R(g)(Z+1)^{-1 / 2}\right\| \leqq \text { const }|g|_{\tau}
$$

where $|g|_{\tau}=\left(\int_{E_{d}}|g(k)|^{2} \mu(k)^{\tau} d k\right)^{1 / 2}$ and the constant depends only on $\mu_{0}$ and $\tau$. For in fact if we put $a(g)=\int g(k) a(k) d k$ then for any $\psi$ in $\mathscr{E}_{1}$ 
we have $\left\|a(g)^{*} \psi\right\|^{2}=\|a(g) \psi\|^{2}+|g|_{0}^{2}\|\psi\|^{2}$ so that $\left\|a(g)^{*} \psi\right\| \leqq\|a(g) \psi\|$ $+|g|_{0}\|\psi\|$ and $\|R(g) \psi\|=\left\|\left(a(g)+a(g)^{*}\right) \psi\right\| \leqq 2\|a(g) \psi\|+|g|_{0}\|\psi\|$. But

$$
\begin{aligned}
\|a(g) \psi\| & =\left\|\int g(k) a(k) \psi d k\right\| \\
& \leqq \int|g(k)|\|a(k) \psi\| d k \\
& \leqq|g|_{\tau}\left(\int \mu(k)^{-\tau}\|a(k) \psi\|^{2} d k\right)^{1 / 2} \\
& =|g|_{\tau}\left\|Z^{1 / 2} \psi\right\| .
\end{aligned}
$$

Since $|g|_{0} \leqq \mu_{0}^{-\tau / 2}|g|_{\tau}$ we have

$$
\begin{aligned}
\|R(g) \psi\| & \leqq \text { const }|g|_{\tau}\left(\left\|Z^{1 / 2} \psi\right\|+\|\psi\|\right) \\
& \leqq \text { const }|g|_{\tau}\left\|(Z+1)^{1 / 2} \psi\right\| .
\end{aligned}
$$

Replacing $\psi$ by $(Z+1)^{-1 / 2} \psi$ (which is again in $\mathscr{E}_{1}$ ) yields (4.24) (cf. [7] on $N_{\tau}$ estimates).

Combining (4.18), (4.21)-(4.24), (4.15), and (3.5) we have

$$
\begin{aligned}
& \left\|\left(e^{i t S\left(g_{n}\right)}-e^{i t S\left(g_{m}\right)}\right) u \otimes \sigma(A) \psi_{0}\right\| \\
& \leqq\|A\| \lim _{j} \sup \left\|\left(e^{i t R\left(g_{n}-g_{m}\right)}-I\right) u \otimes \psi_{j}\right\| \\
& \leqq \text { const }\|A\| T\left|g_{n}-g_{m}\right|_{\tau} \limsup _{j}\left\|(Z+1)^{1 / 2} u \otimes \psi_{j}\right\| \\
& \leqq \text { const }\|A\| T\left|g_{n}-g_{m}\right|_{\tau}\|u\| \lim _{j} \sup \left(\int \mu(k)^{-\tau}\left|f_{j}(k) / \mu(k)\right|^{2} d k\right)^{1 / 2} .
\end{aligned}
$$

The last factor is finite by an assumption of the theorem while $\left|g_{n}-g_{m}\right|_{\tau} \rightarrow 0$ as $n$ and $m \rightarrow \infty$. Hence the left side of (4.25) goes to zero uniformly for $|t| \leqq T$ as $n$ and $m$ go to infinity. This proves the existence of a well defined self-adjoint operator $S(g)$. We note incidentally that taking the limit on $n$ in (4.25) yields

$$
\left\|\left(e^{i t S(g)}-e^{i t S\left(g_{m}\right)}\right) u \otimes \sigma(A) \psi_{0}\right\| \leqq C\left|g-g_{m}\right|_{\tau}
$$

for all $m$ such that $B \subset\{k:|k| \leqq m\}$ where the constant $C$ does not depend on $g$ or $m$.

In order to prove strong continuity of the map $g \rightarrow e^{i t S(g)}$ we consider a sequence $h_{j}$ in $K_{\tau}$ which converges in $K_{\tau}$ norm to $h$. It suffices to prove $e^{i t S\left(h_{j}\right)} \varphi$ converges strongly to $e^{i t S(h)} \varphi$ when $\varphi=u \otimes \sigma(A) \psi_{0}$ is an element of the form considered above since finite linear combinations of these vectors are dense in $\mathscr{K}_{p h}$. Let $\varepsilon>0$. Choose $m$ so large that $B \subset\{k:|k| \leqq m\}$ and such that $C\left(\int_{|k|>m}|h(k)|^{2} \mu(k)^{\tau} d k\right)^{1 / 2} \leqq \varepsilon$, where $C$ is the constant appearing in (4.26). Put $h_{j}=v_{j}+w_{j}$ and $h=v+w$ where $v_{j}(k)=h_{j}(k)$ for $|k| \leqq m$ and is zero otherwise and $v(k)=h(k)$ for $|k| \leqq m$ and is zero otherwise. Then since $C|w|_{\tau} \leqq \varepsilon$ and $\left|w-w_{j}\right|_{\tau} \rightarrow 0$ we have $C\left|w_{j}\right|_{\tau} \leqq 2 \varepsilon$ for 
all sufficiently large $j$. It follows from (4.26) that

$$
\begin{aligned}
\left\|\left(e^{i t S\left(h_{j}\right)}-e^{i t S(h)}\right) \varphi\right\| \leqq\left\|\left(e^{i t S\left(h_{j}\right)}-e^{i t S\left(v_{j}\right)}\right) \varphi\right\| \\
+\left\|\left(e^{i t S\left(v_{j}\right)}-e^{i t S(v)}\right) \varphi\right\|+\left\|\left(e^{i t S(v)}-e^{i t S(h)}\right) \varphi\right\| \\
\quad \leqq 3 \varepsilon+\left\|\left(e^{i t S\left(v_{j}\right)}-e^{i t S(v)}\right) \varphi\right\|
\end{aligned}
$$

for all sufficiently large $j$ and for $|t| \leqq T$. Now $\left|v_{j}-v\right|_{\tau} \rightarrow 0$ so $v_{j}$ converges to $v$ in $L^{2}\left(E_{d}, d k\right)$. It is known [11] that the map $g \rightarrow e^{i t R(g)}$ is a strongly continuous map from $L^{2}\left(E_{d}, d k\right)$ and since $e^{i t S\left(v_{k}\right)}=\hat{\sigma}\left(e^{i t R\left(v_{k}\right)}\right)$ and $\hat{\sigma}$ is unitarily implementable on $\mathscr{C}(\{k:|k| \leqq m\})$ by Corollary 1.2 , it follows that the last term on the right of (4.27) goes to zero as $j \rightarrow \infty$. This establishes the strong continuity of the map $g \rightarrow e^{i t S(g)}$.

The uniqueness of $S(g)$ follows immediately from i) and ii) since ii) determines $S(g)$ uniquely when $g$ has compact support and i) determines $e^{i t S(g)}$ uniquely for each $t$ for all $g$ in $K_{\tau}$ because the functions in $K_{\tau}$ with compact support are dense in $K_{\tau}$.

The validity of (2.15) for all $g$ and $g^{\prime}$ in $K_{\tau}$ follows from the fact that both sides are strongly (jointly) continuous functions of $g$ and $g^{\prime}$ and the equality is valid, as has already been observed, when $g$ and $g^{\prime}$ have compact support.

The proof of part b) is similar to that of part a) and somewhat simpler. If $h$ is a bounded measurable real valued function on $E_{d}$ with compact support then $e^{i t D_{h}}$ lies in $\mathscr{C}(B)$ for some bounded open set $B$ by Proposition 2.2 and by Corollary $1.2 \mathrm{~b}$ ) or c) and Stone's theorem the equation iv) determines a unique self-adjoint operator $N(h)$ on $\mathscr{K}_{p h}$. The operators $N(h)$ commute with each other because the operators $D_{h}$ do and $\hat{\sigma}$ is an isomorphism. If now $h$ is any element of $L_{\tau}$ let $h_{n}(k)=h(k)$ if $|k| \leqq n$ and be zero otherwise. We show that $e^{i t N\left(h_{n}\right)}$ converges strongly on $\mathscr{K}_{p h}$ and uniformly with respect to $t$ on bounded $t$ sets. Since

$$
e^{i t N\left(h_{n}\right)}-e^{i t N\left(h_{m}\right)}=e^{i t N\left(h_{m}\right)}\left(e^{i t N\left(h_{n}-h_{m}\right)}-I\right)
$$

(because this identity is valid for the operators $D_{h}$ ) it suffices, as before, to show that $\lim _{n, m \rightarrow \infty}\left\|\left(e^{i t N\left(h_{n}-h_{m}\right)}-I\right) u \otimes \psi_{0}\right\|=0$ uniformly for $|t| \leqq T$.

For this purpose we need only show that $\left\|\left(e^{i t\left(D_{h_{n}}-h_{m}\right)}-I\right) u \otimes \psi_{j}\right\|$ goes to zero uniformly in $j$ and $|t| \leqq T$ as $n, m \rightarrow \infty$, as noted before. But by Proposition 3.3 and Eq. (4.15) we have

$$
\begin{aligned}
\left\|\left(e^{i t D_{h_{n}}-h_{m}}-I\right) u \otimes \psi_{j}\right\| & \leqq \int_{0}^{|t|}\left\|D_{h_{n}-h_{m}} u \otimes \psi_{j}\right\| d s \\
& \leqq T\|u\|\left\|B_{h_{n}-h_{m}} \psi_{j}\right\| .
\end{aligned}
$$

Since

$$
B_{h}^{2} \psi_{j}=B_{v} \psi_{j}+B_{h^{2}} \psi_{j}
$$


where $v\left(k_{1}, k_{2}\right)=h\left(k_{1}\right) h\left(k_{2}\right)$ (see Remark 3.2 for notation) it follows from (3.5) that

$$
\begin{aligned}
\left\|B_{h_{n}-h_{m}} \psi_{j}\right\|^{2} \leqq & \left(\int\left|h_{n}(k)-h_{m}(k)\right|\left|f_{j}(k) / \mu(k)\right|^{2} d k\right)^{2} \\
& +\int\left|h_{n}(k)-h_{m}(k)\right|^{2}\left|f_{j}(k) / \mu(k)\right|^{2} d k \\
\leqq & \operatorname{const}\left(\int\left|h_{n}(k)-h_{m}(k)\right| \mu(k)^{\tau}\left\{\mu(k)^{-\tau-3}\right\} d k\right)^{2} \\
& +\operatorname{const} \int\left|h_{n}(k)-h_{m}(k)\right|^{2} \mu(k)^{\tau}\left\{\mu(k)^{-\tau-3}\right\} d k .
\end{aligned}
$$

The last two integrals converge to zero by the dominated convergence theorem since the expressions in braces are integrable and the other factor under each integral converges to zero pointwise and boundedly. This establishes the existence of self-adjoint operators $N(h)$. We note that it follows from the preceding inequalities that

$$
\begin{aligned}
& \left\|\left(e^{i t N(h)}-e^{i t N\left(h_{m}\right)}\right) u \otimes \sigma(A) \psi_{0}\right\| \\
& \leqq
\end{aligned}
$$

where $C_{1}$ and $C_{2}$ are constants. The proof that $e^{i t N(h)}$ is a strongly continuous function of $h$ in the indicated topology now follows from (4.29) in exactly the same way as i) followed from (4.26). This establishes properties iii) and iv) for the operators $N(h)$ and uniqueness follows from these properties because the functions of compact support in $L_{\tau}$ are dense in $L_{\tau}$ in the indicated topology.

The linearity, commutativity and order preserving character of the operators $N(h)$ as well as the Eq. (2.16) follow for functions with compact support from the corresponding properties for the operators $D_{h}$ because $\sigma$ is unitarily implementable on each algebra $\mathscr{C}(B)$. For general $h$ and $h^{\prime}$ in $L_{\tau}$ and $g$ in $K_{\tau}$ and real $\alpha$ and $\beta$ the equation $e^{i t N\left(\alpha h+\beta h^{\prime}\right)}=e^{i t \alpha N(h)} e^{i t \beta N\left(h^{\prime}\right)}$ follows by taking strong limits as $h$ and $h^{\prime}$ are approximated by functions with compact support. This establishes the commutativity of $N(h)$ and $N\left(h^{\prime}\right)$ as well as the linearity. The Eq. (2.16) also follows by taking strong limits over approximations to $h$ and $g$ by functions of compact support. It remains to prove that $N(h) \geqq 0$ if $h \geqq 0$ and $h$ is in $L_{\tau}$. If $h_{n}(k)=h(k)$ for $|k| \leqq n$ and zero otherwise then $N\left(h_{n}\right) \geqq 0$. If $f$ is a $C^{\infty}$ function on the line with compact support in $(-\infty, 0)$ and $\hat{f}$ is its Fourier transform then for any vectors $\varphi$ and $\psi$ in $\mathscr{K}_{p h}$

$$
\begin{aligned}
(f(N(h)) \varphi, \psi) & =(2 \pi)^{-1} \int_{-\infty}^{\infty}\left(e^{-i t N(h)} \varphi, \psi\right) \hat{f}(t) d t \\
& =\lim _{n \rightarrow \infty}(2 \pi)^{-1} \int_{-\infty}^{\infty}\left(e^{i t N\left(h_{n}\right)} \varphi, \psi\right) \hat{f}(t) d t \\
& =\lim _{n \rightarrow \infty}\left(f\left(N\left(h_{n}\right)\right) \varphi, \psi\right) \\
& =0
\end{aligned}
$$


since $f\left(N\left(h_{n}\right)\right)=0$ for each $n$. Hence $f(N(h))=0$ and therefore $N(h) \geqq 0$. This concludes the proof of Theorem 2 .

Proof of Corollary 2.1. If $A$ is a self-adjoint operator on a Hilbert space then a vector $v$ is in $\mathscr{D}(A)$ if $\left\|t^{-1}\left(e^{i t A}-1\right) \psi\right\|$ is uniformly bounded for $t \neq 0$. This follows from the spectral theorem and the equation

$$
\int_{-\infty}^{\infty} \lambda^{2} d\left(E_{\lambda} v, v\right)=\lim _{n \rightarrow \infty} \lim _{t \rightarrow 0} \int_{-n}^{n}\left|\frac{e^{i t \lambda}-1}{t}\right|^{2} d\left(E_{\lambda} v, v\right)
$$

where $E(\cdot)$ is the spectral resolution of $A$. If $h$ has compact support then, in virtue of (4.19), the equation $e^{i t D_{h}}=I \otimes_{m} e^{i t B_{h}}$, and Eq. (4.28), as well as Proposition 3.3, we have

$$
\begin{aligned}
&\left\|t^{-1}\left(e^{i t N(h)}-1\right) u \otimes \psi_{0}\right\|^{2}=\limsup _{j}\left\|t^{-1}\left(e^{i t D_{h}}-1\right) u \bigotimes_{m} \psi_{j}\right\|^{2} \\
&= \lim \sup _{j}\|u\|^{2}\left\|t^{-1}\left(e^{i t B_{h}}-1\right) \psi_{j}\right\|^{2} \\
& \leqq \lim \sup _{j}\|u\|^{2}\left\|B_{h} \psi_{j}\right\|^{2} \\
& \leqq\|u\|^{2} \limsup _{j}\left\{\left(\int|h(k)|\left(f_{j}(k) / \mu(k)\right)^{2} d k\right)^{2}\right. \\
&\left.+\int h(k)^{2}\left(f_{j}(k) / \mu(k)\right)^{2} d k\right\} \\
& \leqq \mathrm{const}\|u\|^{2}\left\{\left(\int|h(k)| \mu(k)^{-3} d k\right)^{2}+\int h(k)^{2} \mu(k)^{-3} d k\right\} .
\end{aligned}
$$

If $h$ is a general element in $L_{\tau}$ and $h_{n}(k)=h(k)$ for $|k| \leqq n$ and is zero otherwise, then (4.31) applies to $h_{n}$, and in view of Theorem 2 (iii) and the dominated convergence theorem we may take the limit as $n \rightarrow \infty$ and obtain the validity of (4.31) for any $h$ in $L_{\tau}$. This establishes the corollary.

The next two lemmas are preparatory to the proof of Theorem 3 . We recall that $H_{n}(p)$ denotes the restriction of the total Hamiltonian $H_{n}$ with cutoff function $f_{n}$ to the infinitesimal subspace of total momentum $p$.

Lemma 4.3. Let $P=\left(P_{1}, \ldots, P_{d}\right)$ denote the vector of momentum operators for the Boson field [cf. Eq. (2.5)] and let $E(P)=\left(m^{2}+P^{2}\right)^{1 / 2}$. Let $S$ be a bounded open set in $E_{d}$ and let $A$ be in $\mathscr{A}(S)$. Denote by $P^{\prime}=\left(P_{1}^{\prime}, \ldots, P_{d}^{\prime}\right)$ the vector of self-adjoint operators given by

$$
P_{j}^{\prime}=\int_{k \in S} k_{j} b(k)^{*} b(k) d k
$$

where $k=\left(k_{1}, \ldots, k_{d}\right)$. Suppose that $\psi$ and $A \psi$ are in the domains of $P_{j}, P_{j}^{\prime}$ and $\left(P_{j}^{\prime}\right)^{2}$ for $j=1, \ldots, d$. Then $\psi$ is in the domain of $[E(P), A]$ and

$$
\begin{aligned}
\|[E(P), A] \psi\| \leqq & \frac{1}{2 m}\left\{\left\|\left(P^{\prime}\right)^{2} A \psi\right\|+\|A\|\left\|\left(P^{\prime}\right)^{2} \psi\right\|\right\} \\
& +2 \sum_{j=1}^{d}\left\{\left\|P_{j}^{\prime} A \psi\right\|+\|A\|\left\|P_{j}^{\prime} \psi\right\|\right\}
\end{aligned}
$$

where $\left(P^{\prime}\right)^{2}=\sum_{j=1}^{d}\left(P_{j}^{\prime}\right)^{2}$. 
Proof. Since $E(p) \leqq m+\left|p_{1}\right|+\cdots+\left|p_{d}\right|$ for all real $p_{1}, \ldots, p_{d}$, and $P_{1}, \ldots, P_{d}$ commute it follows that $\psi$ and $A \psi$ are in the domain of $E(P)$ and hence $\psi$ is in the domain of $[E(P), A]$. Let

$$
Q_{j}=\int_{k \notin S} k_{j} b(k)^{*} b(k) d k \quad j=1, \ldots, d .
$$

If $B, C$, and $D$ are three self-adjoint operators with a common core $\mathscr{E}$ then the equation $B \varphi+C \varphi=D \varphi$ for all $\varphi$ in $\mathscr{E}$ implies that $D$ is the closure of $B+C$. For denoting closure by a bar and restriction to $\mathscr{E}$ by a subscript $e$ we have

while

$$
(A+B)^{-} \supset\left(A_{e}+B_{e}\right)^{-}=\bar{C}_{e}=C
$$

so that

$$
(A+B)^{*} \supset A^{*}+B^{*}=A_{e}^{*}+B_{e}^{*} \supset A_{e}+B_{e}=C_{e}
$$

$$
(A+B)^{-} \subset C_{e}^{*}=C \text {. }
$$

Thus since $P_{j}=P_{j}^{\prime}+Q_{j}$ on $\mathscr{E}, P_{j}$ is the closure of $P_{j}^{\prime}+Q_{j}$ and $Q_{j}$ is the closure of $P_{j}-P_{j}^{\prime}$. In particular $\psi$ and $A \psi$ are in the domain of $Q_{j}$, $j=1, \ldots, d$ and hence in the domain of $E(Q)$. Since $A$ is in $\mathscr{A}(S)$ it commutes with any function of the $Q_{j}$. In particular $[E(Q), A] \psi=0$. Therefore

$$
[E(P), A] \psi=[E(P)-E(Q), A] \psi .
$$

Now if $p, p^{\prime}$, and $q$ are vectors in $E_{d}$ with $p=p^{\prime}+q$ then

$$
\begin{aligned}
E(p)-E(q) & =\frac{\left(m^{2}+\left|p^{\prime}+q\right|^{2}\right)-\left(m^{2}+|q|^{2}\right)}{E(p)+E(q)} \\
& =(E(p)+E(q))^{-1}\left|p^{\prime}\right|^{2}+\sum_{j=1}^{d} 2 q_{j}(E(p)+E(q))^{-1} p_{j}^{\prime} .
\end{aligned}
$$

Since the operators $P_{j}, P_{j}^{\prime}, Q_{j}$ are commuting self-adjoint operators with $P_{j}=$ closure of $P_{j}^{\prime}+Q_{j}$ and since $\left\|(E(P)+E(Q))^{-1}\right\| \leqq(2 m)^{-1}$ while $\left\|Q_{j}(E(P)+E(Q))^{-1}\right\| \leqq 1$ it follows that

$$
\begin{aligned}
\|[E(P), A] \psi\|= & \|[E(P)-E(Q), A] \psi\| \\
\leqq & \|(E(P)-E(Q)) A \psi\|+\|A(E(P)-E(Q)) \psi\| \\
\leqq & (2 m)^{-1}\left\|\left(P^{\prime}\right)^{2} A \psi\right\|+2 \sum_{j}\left\|P_{j}^{\prime} A \psi\right\| \\
& +\|A\|(2 m)^{-1}\left\|\left(P^{\prime}\right)^{2} \psi\right\|+2\|A\| \sum_{j=1}^{d}\left\|P_{j}^{\prime} \psi\right\| .
\end{aligned}
$$

This proves the Lemma.

The next lemma was first proved rigorously in [3, Theorem 1]. We give a short proof. If $U$ is any unitary operator on the one Boson Hilbert space $\mathscr{H}=L^{2}\left(E_{d}\right)$ then, regarding $\mathscr{F}$ as the space of all symmetric tensors over $\mathscr{H}$, we denote by $\Gamma(U)$ the unitary operator on $\mathscr{F}$ which is 
$U \otimes U \otimes \cdots \otimes U$ on symmetric $n$-tensors for $n \geqq 1$ and is the identity on zero rank tensors. If $D$ is a self-adjoint operator on $\mathscr{H}$ then $d \Gamma(D)$ denotes the self-adjoint operator on $\mathscr{F}$ defined via Stone's theorem by the equation $\Gamma\left(e^{i t D}\right)=\exp (i t d \Gamma(D))$ for all real numbers $t . R_{0}(g)$ is defined by Eq. (2.6).

Lemma 4.4. Let $D$ be a self-adjoint operator on $\mathscr{H}$ and let $g$ be in the domain of $D$. If $\psi$ is in $\mathscr{D}(d \Gamma(D)) \cap \mathscr{D}\left(N^{1 / 2}\right)$ where $N$ is the number operator on $\mathscr{F}$ then $\psi$ is in the domain of $d \Gamma(D) e^{i R_{0}(g)}$ and

$$
d \Gamma(D) e^{i R_{0}(g)} \psi=e^{i R_{0}(g)}\left\{d \Gamma(D) \psi+(D g, g) \psi+R_{0}(i D g) \psi\right\} .
$$

Proof. We start with a well known and simple to prove identity: $\Gamma(U) R_{0}(g) \Gamma(U)^{-1}=R_{0}(U g)$ [2, Theorem 4] which by the functional calculus yields $\Gamma(U) \exp \left(i R_{0}(g)\right)=\exp \left(i R_{0}(U g)\right) \Gamma(U)$. Substituting $U=e^{i t D}$ and using the commutation relation

$$
e^{i R_{0}(U g)}=e^{i R_{0}(g)} e^{i R_{0}((U-I) g)} e^{i \operatorname{Im}(U g, g)}
$$

(cf. 2.15) we obtain

$$
\Gamma\left(e^{i t D}\right) e^{i R_{0}(g)} \psi=e^{i R_{0}(g)} e^{i R_{0}\left(e^{i t D} g-g\right)} e^{i \operatorname{Im}\left(e^{i t D g, g)}\right.} \Gamma\left(e^{i t D}\right) \psi .
$$

By Stone's theorem the lemma will be proved if we can show that the derivative of the right side exists at $t=0$ and takes the appropriate value. Thus it clearly suffices to prove that the derivative of $e^{i R_{0}\left(e^{i t D g-g)}\right.} \psi$ exists at $t=0$ and equals $i R_{0}(i D g) \psi$. But, using (2.15) again, we have

$$
\begin{gathered}
e^{i R_{0}\left(e^{i t D} g-g\right)} \psi=e^{i R_{0}\left(\left(e^{i t D-I-i t D) g)}\right.\right.} e^{i R_{0}(i t D g)} \\
\cdot e^{-i \operatorname{Im}\left(\left(e^{i t D}-I-i t D\right) g, i t D g\right)} \psi .
\end{gathered}
$$

The assertion now follows from the inequality

$$
\begin{gathered}
\left\|t^{-1}\left(\exp \left[i R_{0}\left(\left(e^{i t D}-I-i t D\right) g\right)\right]-I\right) \psi\right\| \leqq\left\|t^{-1} R_{0}\left(\left(e^{i t D}-I-i t D\right) g\right) \psi\right\| \\
\leqq\left\|t^{-1}\left(e^{i t D}-I\right) g-i D g\right\|\left\|(N+1)^{1 / 2} \psi\right\|
\end{gathered}
$$

which goes to zero as $t \rightarrow 0$, showing that the derivative of $\exp \left[i R_{0}\left(\left(e^{i t D}-I-i t D\right) g\right)\right] \varphi$ is zero at $t=0$. This proves the lemma.

Remark 4.1. The lemma implies that if $B=d \Gamma(D)+(D g, g) I+R_{0}(i D g)$ then the closure $\bar{B}$ of $B$ equals $e^{-i R_{0}(g)} d \Gamma(D) e^{i R_{0}(g)}$, which is the assertion of Theorem 1 of [3]. For any vector $\psi$ in $\mathscr{D}(B)$ can be approximated in $B$ graph norm by vectors in $\mathscr{D}\left(N^{1 / 2}\right) \cap \mathscr{D}(d \Gamma(D))$ (e.g. by finite particle vectors) so that (4.33) implies $\bar{B} C e^{-i R_{0}(g)} d \Gamma(D) e^{i R_{0}(g)}$. However by the spectral theorem we may write $D=D_{1}+D_{2}$ where $D_{1}$ and $D_{2}$ are commuting self-adjoint operators with $D_{1} \geqq I, D_{2} \leqq-I, g \in \mathscr{D}\left(D_{1}\right) \cap \mathscr{D}\left(D_{2}\right)$ and $d \Gamma(D)=$ closure of $\left(d \Gamma\left(D_{1}\right)+d \Gamma\left(D_{2}\right)\right)$. The corresponding operators $B_{1}$ and $B_{2}$ are self-adjoint on their domains since $R_{0}\left(i D_{j} g\right)$ is infinitesimal 
with respect to $d \Gamma\left(D_{j}\right), j=1,2$, and so $B_{j}=e^{-i R_{0}(g)} d \Gamma\left(D_{j}\right) e^{i R_{0}(g)}, j=1,2$. Since $B_{1}+B_{2} C B$ we have $e^{-i R_{0}(g)}\left(d \Gamma\left(D_{1}\right)+d \Gamma\left(D_{2}\right)\right) e^{i R_{0}(g)}=B_{1}+B_{2} C B$. Hence $e^{-i R_{0}(g)} d \Gamma(D) e^{i R_{0}(g)} \subset \bar{B}$.

Lemma 4.5. If $f_{n}$ satisfies the hypotheses of Theorem 2 and $\psi_{n}$ denotes the rest state of $H_{n}$ with unit norm then for each operator $A$ in $\mathscr{A}_{0}$ there is a real number $c(A)$ such that

$$
\left\|H_{n}(0) A \psi_{n}\right\| \leqq c(A) \quad \text { for all } n .
$$

Proof. Since $H_{n}(0) \psi_{n}=m_{0} \psi_{n}$ we may write $H_{n}(0) A \psi_{n}=\left[H_{n}(0), A\right] \psi_{n}$ $+m_{0} A \psi_{n}$ provided $A \psi_{n}$ is in the domain of $H_{n}(0)$ and we shall show this in the course of the proof. We will thus obtain

$$
\left\|H_{n}(0) A \psi_{n}\right\| \leqq\left\|\left[H_{n}(0), A\right] \psi_{n}\right\|+m_{0}\|A\| .
$$

Since $A$ is a finite linear combination of operators of the form $e^{i R_{0}(g)}$ where $g$ has compact support it suffices to prove the lemma in case $A=e^{i R_{0}(g)}$ when $g$ is an $L^{2}$ function with support in a bounded open set $S$. We prove that each of the three terms in $\left[H_{n}(0), A\right]$ corresponding to the three terms in (2.4) gives a uniformly bounded contribution to the right side of inequality (4.36). First we consider the middle term of (2.4). This term, in the notation of Lemma 4.4, is $d \Gamma(D)$ where $D$ is the self-adjoint operator of multiplication by $\mu(k)$ on $L^{2}\left(E_{d}\right)$.

Since $\psi_{n}$ is in the domain of $H_{0}$ we may apply Lemma 4.4 to obtain

where

$$
\begin{aligned}
& \left\|\left[\int_{E_{d}} \mu(k) b(k)^{*} b(k) d k, e^{i R_{0}(g)}\right] \psi_{n}\right\| \\
& =\| e^{i R_{0}(g)}\left\{(\mu g, g) \psi_{n}+R_{0}(i \mu g) \psi_{n} \|\right. \\
& \leqq(\mu g, g)+\left\|R_{0}(i \mu g) \psi_{n}\right\| \\
& \leqq(\mu g, g)+2\|\mu g\|\left\|\left(N_{S}+1\right)^{1 / 2} \psi_{n}\right\| \\
& N_{S}=\int_{S} b(k)^{*} b(k) d k .
\end{aligned}
$$

Proposition 3.3 shows that

$$
\begin{aligned}
\left\|\left(N_{S}+1\right)^{1 / 2} \psi_{n}\right\|^{2} & =\left(N_{S} \psi_{n}, \psi_{n}\right)+1 \\
& \leqq \int_{S}\left|f_{n}(k) / \mu(k)\right|^{2} d k+1
\end{aligned}
$$

which is uniformly bounded in $n$.

Next we consider the first term in (2.4). We must show that $\left\|\left[E(P), e^{i R_{0}(g)}\right] \psi_{n}\right\|$ is uniformly bounded in $n$. Putting $P_{j}^{\prime}=\int_{S} k_{j} b(k)^{*} b(k) d k$, $j=1, \ldots, d$, we note first that in view of the proof of Proposition 2.1, in particular the equation $s(m) \leqq m$, we have $m \geqq m_{0}>0$ so that by inequality (4.32) it suffices to show that $\left\|\left(P_{j}^{\prime}\right)^{2} e^{i R_{0}(g)} \psi_{n}\right\|,\left\|P_{j}^{\prime} e^{i R_{0}(g)} \psi_{n}\right\|,\left\|\left(P_{j}^{\prime}\right)^{2} \psi_{n}\right\|$ 
and $\left\|P_{j}^{\prime} \psi_{n}\right\|$ remain uniformly bounded as $n \rightarrow \infty$ for each $j$. (Actually $m \rightarrow \infty$ in all interesting cases.) In the notation of Lemma $4.4 P_{j}^{\prime}$ is $d \Gamma(D)$ where $D$ is multiplication by the bounded function $k_{j} \chi_{s}(k)$ and $\chi_{s}$ is the characteristic function of $S$. Hence by Lemma 4.4

$$
\begin{aligned}
\left\|\left(P_{j}^{\prime}\right)^{a} e^{i R_{0}(g)} \psi_{n}\right\| & =\left\|e^{i R_{0}(g)}\left\{P_{j}^{\prime}+\left(k_{j} g, g\right) I+R_{0}\left(i k_{j} g\right)\right\}^{a} \psi_{n}\right\| \\
& =\left\|\left\{P_{j}^{\prime}+\left(k_{j} g, g\right) I+R_{0}\left(i k_{j} g\right)\right\}^{a} \psi_{n}\right\|
\end{aligned}
$$

for $a=1$ or 2 . All of the operators appearing are dominated by a power of $\left(N_{S}+1\right)^{1 / 2}$ in the following specific sense:

where

$$
\begin{gathered}
\left\|P_{j}^{\prime} \psi\right\| \leqq b\left\|\left(N_{S}+1\right) \psi\right\| \\
b=\sup \left\{\left|k_{j}\right|: k \in S\right\} \\
\left\|\left(P_{j}^{\prime}\right)^{2} \psi\right\| \leqq b^{2}\left\|\left(N_{S}+1\right)^{2} \psi\right\| \\
\left\|P_{j}^{\prime} R_{0}\left(i k_{j} g\right) \psi\right\| \leqq 2 b\left\|k_{j} g\right\|\left\|\left(N_{S}+1\right)^{3 / 2} \psi\right\| \\
\left\|R_{0}\left(i k_{j} g\right)^{2} \psi\right\| \leqq 8\left\|k_{j} g\right\|^{2}\left\|\left(N_{S}+1\right) \psi\right\| .
\end{gathered}
$$

Thus for some constant $C$ independent of $n$ we have

$$
\begin{aligned}
\left\|\left[E(P), e^{i R_{0}(g)}\right] \psi_{n}\right\| & \leqq C\left\|\left(N_{S}+1\right)^{2} \psi_{n}\right\| \\
& \leqq 2 C\left\|e^{N_{S}} \psi_{n}\right\| \\
& \leqq 2 C \exp \left\{\int_{S}\left(e^{2}-1\right)\left|f_{n}(k) / \mu(k)\right|^{2} d k\right\}
\end{aligned}
$$

by Corollary 3.5. This is uniformly bounded in view of the assumptions on the sequence $f_{n}$. We remark that $\left\|\left(N_{S}+1\right)^{2} \psi_{n}\right\|$ could also be estimated easily using just Proposition 3.3 and the commutation relations for the $b(k)$.

Finally the potential $V_{n}$ in $\mathscr{F}_{0}$ is $V_{n}=R_{0}\left(f_{n}\right)$ and the commutation relations show that

Hence

$$
\left[V_{n}, e^{i R_{0}(g)}\right]=-2 i \operatorname{Im}\left(g, f_{n}\right) e^{i R_{0}(g)} .
$$

$$
\left\|\left[V_{n}, e^{i R_{0}(g)}\right] \psi_{n}\right\| \leqq 2\left|\operatorname{Im}\left(g, f_{n}\right)\right| .
$$

Since $g$ has compact support the right side is uniformly bounded in $n$. Thus we have shown that each term on the right of the inequality

$$
\begin{aligned}
\left\|\left[H_{n}(0), A\right] \psi_{n}\right\| \leqq\left\|[E(P), A] \psi_{n}\right\| & +\left\|\left[\int_{E_{d}} \mu(k) b(k)^{*} b(k) d k, A\right] \psi_{n}\right\| \\
& +\left\|\left[V_{n}, A\right] \psi_{n}\right\|
\end{aligned}
$$

is uniformly bounded in $n$ when $A=e^{i R_{0}(g)}$. This concludes the proof of the lemma. 
All tensor products in the remainder of the paper will refer to the total momentum decomposition (2.3) and we shall henceforth write $\otimes$ instead of $\otimes_{m}$.

Lemma 4.6. If $f_{n}$ satisfies the hypotheses of Theorem 2 and $\psi_{n}$ denotes the rest state of $H_{n}$ with unit norm then, for each operator $A$ in $\mathscr{A}_{0}$ and each function $u$ in $L^{2}\left(R_{d}\right)$ with compact support in total momentum space $R_{d}$, there is a real number $c(A, u)$ such that

$$
\left\|H_{n}\left(u \otimes A \psi_{n}\right)\right\| \leqq c(A, u) \quad \text { for all } n .
$$

Proof. Keeping in mind the discussion of Eqs. (2.3) and (2.4) we may write

$$
\left\|H_{n}\left(u \otimes A \psi_{n}\right)\right\|^{2}=\int_{R_{d}}|u(p)|^{2}\left\|H_{n}(p) A \psi_{n}\right\|^{2} d p .
$$

But $H_{n}(p)-H_{n}(0)$ is a bounded operator of norm at most $|p|$. Hence, in view of Lemma 4.5, we may write

$$
\begin{aligned}
\left\|H_{n}\left(u \otimes A \psi_{n}\right)\right\|^{2} & \leqq \int|u(p)|^{2}\left\{\left\|H_{n}(0) A \psi_{n}\right\|+|p|\left\|A \psi_{n}\right\|\right\}^{2} d p \\
& \leqq \int|u(p)|^{2}\{c(A)+|p|\|A\|\}^{2} d p
\end{aligned}
$$

which is finite since $u$ has compact support.

Proof of Theorem 3. Let $S_{n}$ be the open ball of radius $n$ in $E_{d}$. Identifying $L^{2}\left(S_{n}\right)$ with functions in $L^{2}\left(E_{d}\right)$ vanishing off $S_{n}$ we let $\mathscr{M}_{n}$ be a countable dense set in $L^{2}\left(S_{n}\right)$ and put $\mathscr{M}=\bigcup_{n} \mathscr{M}_{n}$. Then $\mathscr{M}$ is a countable dense set in $L^{2}\left(E_{d}\right)$.

Given a weak accumulation point $\omega$ of the $\omega_{n}$ choose a subsequence $n_{j}$ as indicated in Corollary 1.1.

If $u, v, g$ and $h$ are in $\mathscr{M}$ then by Lemma $4.6\left(H_{n_{j}}\left(u \otimes e^{i R_{0}(g)} \psi_{n_{j}}\right)\right.$, $\left.v \otimes e^{i R_{0}(h)} \psi_{n_{j}}\right)$ is a bounded sequence. Therefore we may find a convergent subsequence. Since $\mathscr{M} \times \mathscr{M} \times \mathscr{M} \times \mathscr{M}$ is countable there exists, by diagonalization, a sequence $n_{j_{k}} \rightarrow \infty$ such that $\left(H_{n_{j_{k}}}\left(u \otimes e^{i R_{0}(g)} \psi_{n_{j_{k}}}\right)\right.$, $v \otimes e^{i R_{0}(h)} \psi_{n_{j_{k}}}$ ) converges for all $u, v, g$ and $h$ in $\mathscr{M}$. We shall be concerned henceforth only with this subsequence to which we now restrict our consideration.

We therefore change notation, writing $\psi_{j}, \omega_{j}, H_{j}$ and we assume that $\omega_{j}$ converges to $\omega$ in the two senses indicated in Corollary 1.1 while

$$
\left(H_{j}\left(u \otimes e^{i R_{0}(g)} \psi_{j}\right), v \otimes e^{i R_{0}(h)} \psi_{j}\right)
$$

converges whenever $u, v, g, h$ are in $\mathscr{M}$.

We assert that (4.40) converges for all $u, v, g$, and $h$ in $L^{2}\left(E_{d}\right)$ with compact support. In order to see this let $u$ and $g$ be in $\mathscr{M}$ and let $v$ and $h$ be in $L^{2}\left(E_{d}\right)$ with support in $S_{r}$, say. Choose $v_{0}$ and $h_{0}$ in $\mathscr{M}_{r}$ and put 
$w_{j}=H_{j}\left(u \otimes e^{i R_{0}(g)} \psi_{j}\right), \quad \alpha_{j}=v \otimes e^{i R_{0}(h)} \psi_{j} \quad$ and $\quad \beta_{j}=v_{0} \otimes e^{i R_{0}\left(h_{0}\right)} \psi_{j}$. Then since $\left|\left(w_{j}, \alpha_{j}\right)-\left(w_{k}, \alpha_{k}\right)\right| \leqq\left|\left(w_{j}, \alpha_{j}\right)-\left(w_{j}, \beta_{j}\right)\right|+\left|\left(w_{j}, \beta_{j}\right)-\left(w_{k}, \beta_{k}\right)\right|$ $+\left|\left(w_{k}, \beta_{k}\right)-\left(w_{k}, \alpha_{k}\right)\right|$ we may write, estimating $\left\|w_{j}\right\| \leqq$ const by Lemma 4.6,

Hence

$$
\begin{aligned}
\left|\left(w_{j}, \alpha_{j}\right)-\left(w_{k}, \alpha_{k}\right)\right| \leqq & \operatorname{const}\left(\left\|\alpha_{j}-\beta_{j}\right\|+\left\|\alpha_{k}-\beta_{k}\right\|\right) \\
& +\left|\left(w_{j}, \beta_{j}\right)-\left(w_{k}, \beta_{k}\right)\right| .
\end{aligned}
$$

$$
\limsup _{j, k \rightarrow \infty}\left|\left(w_{j}, \alpha_{j}\right)-\left(w_{k}, \alpha_{k}\right)\right| \leqq \text { const } \limsup _{j, k \rightarrow \infty}\left(\left\|\alpha_{j}-\beta_{j}\right\|+\left\|\alpha_{k}-\beta_{k}\right\|\right) .
$$

But $\left\|\alpha_{j}-\beta_{j}\right\| \leqq\left\|v-v_{0}\right\|+\|v\|\left\|\left(e^{i R_{0}(h)}-e^{i R_{0}\left(h_{0}\right)}\right) \psi_{j}\right\|$. By the definition of $\omega$ and $\psi_{0}, \overline{\lim }\left\|\alpha_{j}-\beta_{j}\right\| \leqq\left\|v-v_{0}\right\|+\|v\|\left\|\sigma\left(e^{i R_{0}(h)}-e^{i R_{0}\left(h_{0}\right)}\right) \psi_{0}\right\|$. Now the map $h \rightarrow e^{i R_{0}(h)}$ is strongly continuous on $L^{2}\left(E_{d}\right)$ and by Corollary 1.1 the restriction of $\sigma$ to $\mathscr{A}\left(S_{r}\right)$ is unitarily implementable. Hence the map $h \rightarrow \sigma\left(e^{i R_{0}(h)}\right)$ is strongly continuous on $L^{2}\left(S_{r}\right)$. Therefore if $h_{0}$ is chosen to be sufficiently close to $h$ and in $L^{2}\left(S_{r}\right)$ then $\left\|\sigma\left(e^{i R_{0}(h)}-e^{i R_{0}\left(h_{0}\right)}\right) \psi_{0}\right\|$ can be made arbitrarily small. Choosing $v_{0}$ in $\mathscr{M}_{r}$ such that $\left\|v-v_{0}\right\|$ is small shows that $\limsup _{j, k}\left|\left(w_{j}, \alpha_{j}\right)-\left(w_{k}, \alpha_{k}\right)\right|=0$. This shows that (4.40) converges for all $u$ and $g$ in $\mathscr{M}$ and all $v$ and $h$ in $L^{2}\left(E_{d}\right)$ with compact support. Bringing the self-adjoint operator $H_{j}$ over to the other side of the inner product in (4.40) we may now repeat the preceding argument, holding $v$ and $h$ fixed, to obtain the assertion following (4.40).

Since each operator in $\mathscr{A}_{0}$ is a finite linear combination of the operators $e^{i R_{0}(g)}, g \in L^{2}\left(S_{r}\right)$ it follows that, for any $A$ and $B$ in $\mathscr{A}_{0}$ and $u$ and $v$ of compact support, $\left(H_{j}\left(u \otimes A \psi_{j}\right), v \otimes B \psi_{j}\right)$ converges as $j \rightarrow \infty$.

Denote by $L_{c}^{2}\left(R_{d}\right)$ the space of functions in $L^{2}\left(R_{d}\right)$ with compact support. Let $\mathscr{L}$ be the linear space in $\mathscr{K}_{p h}$ consisting of all vectors $\alpha$ of the form

$$
\alpha=\sum_{k=1}^{n} u_{k} \otimes \sigma\left(A_{k}\right) \psi_{0}
$$

where $u_{k}$ is in $L_{c}^{2}\left(R_{d}\right)$ and $A_{k}$ is in $\mathscr{A}_{0}$ for $k=1, \ldots, n$ and the sum is finite. We assert that $\mathscr{L}$ is dense in $\mathscr{K}_{p h}$. To this end it suffices to show that $\sigma\left(\mathscr{A}_{0}\right) \psi_{0}$ is dense in $\mathscr{K}_{0}$. But for any bounded open set $S \subset E_{d} \mathscr{A}(S)$ is the strong closure of $\mathscr{A}_{0} \cap \mathscr{A}(S)$ and since $\sigma$ is unitarily implementable on $\mathscr{A}(S)$ it follows that the strong closure of $\sigma\left(\mathscr{A}_{0} \cap \mathscr{A}(S)\right)$ is $\sigma(\mathscr{A}(S))$. Thus the closure of $\sigma\left(\mathscr{A}_{0}\right) \psi_{0}$ contains $\sigma(\mathscr{A}(S)) \psi_{0}$ for all bounded open sets $S$. Since $\bigcup_{S} \sigma(\mathscr{A}(S)) \psi_{0}$ is dense in $\mathscr{K}_{0}$ so is $\sigma\left(\mathscr{A}_{0}\right) \psi_{0}$. Therefore $\mathscr{L}$ is dense in $\mathscr{K}_{p h}$.

Let

$$
\beta=\sum_{r=1}^{m} v_{r} \otimes \sigma\left(B_{r}\right) \psi_{0}
$$


be another element in $\mathscr{L}$. We have seen that the limit defining the following sesquilinear form $F$ on the algebraic tensor product $L_{c}^{2}\left(R_{d}\right) \otimes \mathscr{A}_{0}$ exists:

$$
\begin{gathered}
F\left(\sum_{j=1}^{n} u_{j} \otimes A_{j}, \sum_{k=1}^{m} v_{k} \otimes B_{k}\right) \\
=\lim _{j \rightarrow \infty}\left(H_{j}\left(\sum_{k=1}^{n} u_{k} \otimes A_{k} \psi_{j}\right), \sum_{r=1}^{m} v_{r} \otimes B_{r} \psi_{j}\right) .
\end{gathered}
$$

Now we note that

$$
\begin{aligned}
\lim _{j \rightarrow \infty}\left\|\Sigma_{r} v_{r} \otimes B_{r} \psi_{j}\right\|^{2} & =\lim _{j \rightarrow \infty} \sum_{r, s}\left(v_{r}, v_{s}\right)\left(B_{r} \psi_{j}, B_{s} \psi_{j}\right) \\
& =\sum_{r, s}\left(v_{r}, v_{s}\right)\left(\sigma\left(B_{r}\right) \psi_{0}, \sigma\left(B_{s}\right) \psi_{0}\right) \\
& =\|\beta\|^{2} .
\end{aligned}
$$

By Lemma 4.6 there exists a constant $C$ depending on $u_{1}, \ldots, u_{n}, A_{1}, \ldots, A_{n}$ but not on $j$ such that $\left\|H_{j}\left(\sum_{k=1}^{n} u_{k} \otimes A_{k} \psi_{j}\right)\right\| \leqq C$ for all $j$. It follows that

$$
\begin{aligned}
|F(\cdot, \cdot)| & \leqq C \lim _{j \rightarrow \infty}\left\|\sum_{r=1}^{m} v_{r} \otimes B_{r} \psi_{j}\right\| \\
& \leqq C\|\beta\| .
\end{aligned}
$$

Similarly, bringing the operator $H_{j}$ to the other side of the inner product in (4.43) it follows that there is a constant $C^{\prime}$ depending only on $v_{1}, \ldots, B_{m}$ such that

$$
|F(\cdot, \cdot)| \leqq C^{\prime}\|\alpha\|
$$

This inequality shows first that if $v_{1}, \ldots, B_{m}$ are held fixed then $F\left(\cdot, \Sigma v_{r} \otimes B_{r}\right)$ defines a linear functional on $L_{c}^{2}\left(R_{d}\right) \otimes \mathscr{A}_{0}$ whose null space contains the kernel of the map $\Sigma u_{k} \otimes A_{k} \rightarrow \Sigma u_{k} \otimes \sigma\left(A_{k}\right) \psi_{0}=\alpha$, so that $F\left(\cdot, \Sigma v_{r} \otimes B_{r}\right)$ defines a linear functional on $\mathscr{L}$. Secondly it shows that the linear functional is continuous on $\mathscr{L}$.

Similarly the inequality (4.44) shows that if the first argument of $F$ is held fixed in $L_{c}^{2}\left(R_{d}\right) \otimes \mathscr{A}_{0}$ then $F$ defines a continuous anti-linear functional on $\mathscr{L}$. Hence $F$ defines a separately continuous sesquilinear form $\langle\alpha, \beta\rangle$ on $\mathscr{L} \times \mathscr{L}$ given by

$$
\langle\alpha, \beta\rangle=F\left(\sum_{k=1}^{n} u_{k} \otimes A_{k}, \sum_{r=1}^{m} v_{r} \otimes B_{r}\right)
$$

where $\alpha$ and $\beta$ are given by (4.41) and (4.42). 
Since $\langle\cdot, \cdot\rangle$ is separately continuous on $\mathscr{L} \times \mathscr{L}$ there is an operator $H^{\prime}$ on $\mathscr{K}_{p h}$ with domain $\mathscr{L}$ such that

$$
\langle\alpha, \beta\rangle=\left(H^{\prime} \alpha, \beta\right)
$$

for $\alpha$ and $\beta$ in $\mathscr{L}$. It follows from the self-adjointness of the operators $H_{j}$ and Eq. (4.43) that $\langle\alpha, \beta\rangle=\overline{\langle\beta, \alpha\rangle}$. Consequently $H^{\prime}$ is a symmetric operator on $\mathscr{L}$. Moreover, since $H_{j} \geqq m_{0} I$ for all $j$ we have

$$
\begin{aligned}
\langle\alpha, \alpha\rangle & =\lim _{j}\left(H_{j}\left(\Sigma u_{k} \otimes A_{k} \psi_{j}\right), \Sigma u_{k} \otimes A_{k} \psi_{j}\right) \\
& \geqq \lim _{j} m_{0}\left\|\Sigma_{k} u_{k} \otimes A_{k} \psi_{j}\right\|^{2} \\
& \geqq m_{0}\|\alpha\|^{2} .
\end{aligned}
$$

Therefore $H^{\prime} \geqq m_{0} I$. Let $H$ denote the Friedrichs extension of $H^{\prime}$. Then $H$ is self-adjoint and $H \geqq m_{0} I$.

Conclusion i) of Theorem 3 is satisfied. Now taking $A$ equal to the identity operator in Eq. (4.39) we have

$$
\begin{aligned}
\left\|H_{j} u \otimes \psi_{j}\right\|^{2} & =\int_{R_{d}}|u(p)|^{2}\left\|\left(H_{j}(p)-H_{j}(0)+m_{0}\right) \psi_{j}\right\|^{2} d p \\
& \leqq \int_{R_{d}}|u(p)|^{2}\left(\left\|\left(H_{j}(p)-H_{j}(0)\right) \psi_{j}\right\|+m_{0}\right)^{2} d p \\
& \leqq \int|u(p)|^{2}\left(m_{0}+|p|\right)^{2} d p .
\end{aligned}
$$

Thus if $\varepsilon>0$ and $\int|u(p)|^{2} d p=1$ and $u(p)=0$ for $|p|>\varepsilon$ then $\left\|H_{j} u \otimes \psi_{j}\right\|$ $\leqq\left(m_{0}+\varepsilon\right)$. Hence

$$
\left(H_{j} u \otimes \psi_{j}, u \otimes \psi_{j}\right) \leqq m_{0}+\varepsilon \text { for all } j .
$$

It follows that $\left(H^{\prime} u \otimes \psi_{0}, u \otimes \psi_{0}\right) \leqq m_{0}+\varepsilon$ and since $\left\|u \otimes \psi_{0}\right\|=1$ we have inf spectrum $(H) \leqq m_{0}+\varepsilon$. Since $\varepsilon$ is arbitrary, conclusion ii) of the theorem follows.

In order to prove part iii) of the theorem we first note that the operator $D_{h}=\int h(k) a(k)^{*} a(k) d k$ was shown in the proof of Proposition 2.2 to be equal to $I \otimes_{m} B_{h}$ [cf. Eq. (4.15)]. We first restrict our attention to the zero momentum subspace.

Note that if $A$ and $C$ are self-adjoint operators such that $A \geqq 0$, and $\mathscr{D}(C) \supset \mathscr{D}(A)$, and $A-C$ is self-adjoint and bounded below, and $\psi$ is a unit eigenvector of $A-C$ with eigenvalue equal to inf spectrum $(A-C)$, then

$$
C \leqq A+(C \psi, \psi) \text {. }
$$

For $A-C \geqq(A-C \psi, \psi)=(A \psi, \psi)-(C \psi, \psi) \geqq-(C \psi, \psi)$. We apply this with $A=H_{j}(0)$ and $C=B_{h}$. Thus

$$
A-C=\int_{E_{d}}(\mu(k)-h(k)) b(k)^{*} b(k) d k+E(-P)+\varphi_{j}(0)
$$


where $\varphi_{j}(0)=\int f_{j}(k)\left(b(k)+b(k)^{*}\right) d k$ and the mass in $E(-P)$ has been chosen so as to make $m_{0}$ the bottom of the spectrum of $H_{j}(0)$. Since $\mu(k)-h(k)$ is a real and even positive function bounded away from zero we may apply Theorem 8 of [8] to obtain a unit eigenvector $\psi$ for $A-C$ with eigenvalue lying at the bottom of the spectrum of the semi-bounded operator $A-C$. Proposition 3.3 is applicable to yield an estimate of $\left(B_{h} \psi, \psi\right)$ except, of course, that $\mu(k)$ must be replaced by $\mu(k)-h(k)$. Thus (3.5) yields

$$
\left(B_{h} \psi, \psi\right) \leqq \int_{E_{d}} h(k)\left|\frac{f_{j}(k)}{\mu(k)-h(k)}\right|^{2} d k .
$$

Hence inequality (4.46) yields

$$
B_{h} \leqq H_{j}(0)+\int_{E_{d}} h(k)\left|\frac{f_{j}(k)}{\mu(k)-h(k)}\right|^{2} d k .
$$

Now $E(-q) \leqq E(p-q)+|p|$ for all vectors $p$ and $q$ in $E_{d}$. Hence $E(-P)$ $\leqq E(p-P)+|p|$ and consequently $H_{j}(0) \leqq H_{j}(p)+|p|$. Therefore

$$
B_{h} \leqq H_{j}(p)+d_{j}(h)+|p|
$$

where $d_{j}(h)=\int h(k)\left|f_{j}(k) /(\mu(k)-h(k))\right|^{2} d k$.

Suppose that the functions $u_{k}$ in Eq. (4.41) have support in $S_{a}$. Put $w_{j}=\sum_{k=1}^{n} u_{k} \otimes A_{k} \psi_{j}$ and $w_{j}(p)=\sum_{k=1}^{n} u_{k}(p) A_{k} \psi_{j}$. Then

$$
\begin{aligned}
\left(D_{h} w_{j}, w_{j}\right) & =\left(\left(I \otimes_{m} B_{h}\right) w_{j}, w_{j}\right) \\
& =\int_{|p| \leqq a}\left(B_{h} w_{j}(p), w_{j}(p)\right) d p \\
& \leqq \int_{|p| \leqq a}\left(\left(H_{j}(p)+d_{j}(h)+|p|\right) w_{j}(p), w_{j}(p)\right) d p \\
& =\left(\left(H_{j}+d_{j}(h)\right) w_{j}, w_{j}\right)+\int_{|p| \leqq a}|p|\left\|w_{j}\right\|^{2} d p \\
& \leqq\left(\left(H_{j}+d_{j}(h)+a\right) w_{j}, w_{j}\right) .
\end{aligned}
$$

We wish to take the limit $j \rightarrow \infty$ in this inequality to obtain

$$
(N(h) \alpha, \alpha) \leqq\left(\left(H^{\prime}+d(h)+a\right) \alpha, \alpha\right) .
$$

However while the right side of (4.49) does indeed converge to the right side of (4.50) by the definition of $H^{\prime}$ and $d_{j}(h)$, we do not know that the left side converges. Thus we proceed as follows. First restrict $h$ to have compact support (in addition to the previous restrictions) in some bounded open set $S$. Let $\varphi$ be a non-negative, continuous, bounded function on $(-\infty, \infty)$ such that $\varphi(t) \leqq t$ for all $t$ in $[0, \infty)$, and assume further that $\varphi$ is the Fourier transform of an integrable function $\hat{\varphi}$. 
Then $\varphi\left(D_{h}\right) \leqq D_{h}$ and therefore

$$
\left(\varphi\left(D_{h}\right) w_{j}, w_{j}\right) \leqq\left(\left(H_{j}+d(h)+a\right) w_{j}, w_{j}\right) .
$$

Now $\varphi\left(D_{h}\right)=I \otimes_{m} \varphi\left(B_{h}\right)$ is in $\mathscr{C}(S)$ since $\varphi$ is bounded and $h$ has compact support. Thus in view of Eq. (4.19) and the definition of $\alpha$ and $w_{j}$ we have $\lim _{j}\left(\varphi\left(D_{h}\right) w_{j}, w_{j}\right)=\left(\hat{\sigma}\left(\varphi\left(D_{h}\right)\right) \alpha, \alpha\right)$. Since $\hat{\sigma}$ is unitarily implementable on $\mathscr{C}(S)$ by a unitary operator which takes $D_{h}$ to $N(h)$ (cf. Theorem 2 , iv) it follows that $\hat{\sigma}\left(\varphi\left(D_{h}\right)\right)=\varphi(N(h))$. Hence taking the limit $j \rightarrow \infty$ in (4.51) we obtain

$$
(\varphi(N(h)) \alpha, \alpha) \leqq\left(\left(H^{\prime}+d(h)+a\right) \alpha, \alpha\right)
$$

for all functions $\varphi$ of the indicated type. Now we may remove the restriction that $h$ have compact support. For if $h$ merely satisfies the hypothesis of the theorem and $h_{n}(k)=h(k)$ for $|k| \leqq n$ and is zero otherwise then $d\left(h_{n}\right) \rightarrow d(h)$ by dominated convergence, as $n \rightarrow \infty$, and, since $e^{i t N\left(h_{n}\right)}$ converges to $e^{i t N(h)}$ strongly, the equation $\varphi\left(N\left(h_{n}\right)\right) \alpha=\int_{-\infty}^{\infty} e^{i t N\left(h_{n}\right)} \alpha \hat{\varphi}(t) d t$ shows that $\varphi\left(N\left(h_{n}\right)\right)$ converges to $\varphi(N(h))$ strongly. Hence (4.52) holds without a compact support restriction on $h$. Next we shall remove the function $\varphi$.

We note incidentally that by Corollary $2.1, \alpha$ is in the domain of $N(h)$. However we shall not use this fact. The domain of $(H+d(h)+a)^{1 / 2}$ is, by the definition of the Friedrichs extension, the closure of $\mathscr{D}\left(H^{\prime}\right)$ in the norm given by the square root of the right side of inequality (4.52). Since the left side of (4.52) is continuous in this norm (in fact in $\mathscr{K}_{p h}$ norm since $\varphi$ is bounded) the inequality persists for all $\alpha$ in $\mathscr{D}\left(H^{1 / 2}\right) \cap L^{2}\left(S_{a}\right) \otimes \mathscr{K}_{0}$ in the form

$$
\begin{gathered}
\left\|\varphi^{1 / 2}(N(h)) \gamma\right\| \leqq\left\|(H+d(h)+a)^{1 / 2} \gamma\right\|, \\
\gamma \in \mathscr{D}\left(H^{1 / 2}\right) \cap L^{2}\left(S_{a}\right) \otimes \mathscr{K}_{0} .
\end{gathered}
$$

Letting $\varphi$ increase to the function $\varphi(t)=t$ through a pointwise convergent sequence of functions of the indicated type, now shows, with the help of the spectral theorem for $N(h)$ and the monotone convergence theorem, that (2.17) holds. We remark that the subspace $L^{2}\left(S_{a}\right) \otimes \mathscr{K}_{0}$ is invariant under $H$. This is clearly the case for $H^{\prime}$ as follows from (4.43), the form of $H_{j}$ and the definition of $H^{\prime}$. Since $H \subset H^{*}$ and $H^{*}$ leaves $L^{2}\left(S_{a}\right) \otimes \mathscr{K}_{0}$ invariant so does $H$. This concludes the proof of Theorem 3 .

\section{§ 5. Concluding Remarks}

5.1. It is clear from the definition of $\mathscr{K}_{p h}$ that the operators corresponding to the total momentum of polaron plus Boson field are definable as self-adjoint operators on $\mathscr{K}_{p h}$. Newton-Wigner polaron 
position operators also act on $\mathscr{K}_{p h}$. Evidence indicates, however, that the polaron momentum operators and Boson momentum operators are not separately definable on $\mathscr{K}_{p h}$ in any reasonable way. This is in keeping with the current view that certain bare observables should not be definable on the physical Hilbert space.

5.2. We have used Fock space methods in this article but it is illuminating to consider the meaning of some of our inequalities in the Schrödinger representation ( $Q$ space representation) of the zero momentum subspace. The use of Fock space is in many ways analogous to the use of the Fourier transform in the study of partial differential operators. In the Schrödinger representation of the zero momentum subspace the Hamiltonian with cutoff is a second order elliptic differential operator (in infinitely many variables) plus a first order pseudo-differential operator (that comes from the polaron kinetic energy term) plus a multiplication operator (that comes from $H_{I}$ ). The rest state is thus an eigenfunction of an elliptic differential operator, and our basic inequalities amount to estimates on the derivatives (of all orders) of this eigenfunction. In fact our estimates can be used to show that the rest state is, in a certain classical sense, an analytic function in the Schrödinger representation.

5.3. Our treatment of this model is incomplete in many fundamental respects. We do not prove convergence of the states $\omega_{n}$, and in fact we have not even used the fact that the cutoff functions $f_{n}(k)$ converge to $\lambda \mu(k)^{-1 / 2}$ in any crucial way. We do not prove the existence of the renormalized polaron field as an operator from the one polaron physical Hilbert space to the zero polaron physical Hilbert space (which is just Fock space), although I have little doubt of its existence, and some of its properties are easy to conjecture. We do not have a proof of the irreducibility of the physical one polaron Hilbert space under the Boson field and the other relevant operators (total momentum and polaron position). Moreover we do not discuss the many polaron physical Hilbert space at all.

Nevertheless the three problems that we have solved - the norm compactness of states (Theorem 1), the extendability of the physical Boson fields from test functions which have compact support in momentum space to all of the Schwartz space $\mathscr{S}$ (Theorem 2), and the construction of the total Hamiltonian (Theorem 3) - seem to us to be basic to any complete treatment of this model as well as other related models with a momentum divergence.

5.4. The mass renormalization has been carried out in the present model by inserting a bare mass $m=m_{0}+\delta m$ ( $m_{0}=$ physical mass) into the kinetic energy function $\left(m^{2}+|p|^{2}\right)^{1 / 2}$ of the nucleon. $\delta m$ is chosen (depending on the cutoff function) so as to maintain the lower bound of the one nucleon Hamiltonian at the (fixed) physical mass $m_{0}$ as the 
cutoff is removed. This is in keeping with the spirit of mass renormalization in relativistic theories. However the theorems and techniques we have described in this paper are equally applicable with no changes of proof if one accomplishes the mass renormalization by defining the renormalized one nucleon Hamiltonian in the total momentum $p$ subspace as

$$
H(p)=\left(H_{0}\right)_{b}+\left(m_{0}^{2}+(p-P)^{2}\right)^{1 / 2}+V+(\delta m) I
$$

where $\delta m$ is again chosen so as to make $m_{0}$ the lower bound of the spectrum of $H(p=0)$. Fröhlich has pointed out in a note [17] that the first method of renormalizing the model leads in the no cutoff limit to a model whose dynamics is that of the scalar field model. (I wish to thank J. Fröhlich for an advance copy of his work.) His suggestion that the renormalization method (5.1) be used in order to achieve a non-trivial dynamics seems compelling.

\section{Bibliography}

1. Agmon, S.: Lectures on elliptic boundary value problems. New York: D. Van Nostrand Co. Inc. 1965.

2. Cook,J.M.: The mathematics of second quantization. Trans. Am. Math. Soc. 74, 222-245 (1953).

3. Cook, J.M.: Asymptotic properties of a Boson field with given source. Jour. of Mathematical Physics 2, 33-45 (1961).

4. Dixmier, J.: Les algèbres d'opérateurs dans l'espace Hilbertien. Paris: GauthierVillars 1969.

5. Glimm, J., Jaffe, A.: The $\lambda\left(\varphi^{4}\right)_{2}$ quantum field theory without cutoffs. II. The field operators and the approximate vacuum. Ann. Math. 91, 362-401 (1970).

6. Glimm, J., Jaffe, A.: The $\lambda\left(\varphi^{4}\right)_{2}$ quantum field theory without cutoffs. III. The physical vacuum. Acta Math. 125, 203-267 (1970).

7. Glimm, J., Jaffe, A.: Quantum field theory models, in the 1970 Les Houches lectures. C. Dewitt and R. Stora, (Ed.), New York: Gordon and Breach Science Publishers 1971.

8. Gross, L.: Existence and uniqueness of physical ground states. Jour. of Functional Analysis 10, 52-109 (1972).

9. Kato,T.: Perturbation theory for linear operators. Berlin-Heidelberg-New York: Springer 1966.

10. Nelson,E.: Interaction of non relativistic particles with a quantized scalar field. Jour. of Mathematical Physics 5, 1190-1197 (1964).

11. Segal, I. E.: Tensor algebras over Hilbert spaces. I. Trans. Am. Math. Soc. 81, 106-134 (1956).

12. Sloan, A.: The relativistic polaron without cutoffs in two space dimensions. Doctoral Dissertation, Cornell University, 1971.

13. Albeverio, S.: Strong asymptotic convergence of fields and states for physical particles in models of quantum field theory. Princeton preprint, 1971.

14. Albeverio, S.: Scattering theory in a model of quantum fields. II. Helvetica Physica Acta 45, 303-321 (1972). 
15. Eckmann, J. P.: A model with persistent vacuum. Commun. math. Phys. 18, 247-264 (1970).

16. Fröhlich, J.: On the infrared problem in a model of scalar electrons and massless scalar Bosons I. II. ETH Thesis 1972.

17. Fröhlich, J.: A note on a paper of L. Gross. E.T.H. Preprint, 1972.

\section{Gross}

Cornell University

Department of Mathematics

White Hall

Ithaca, N.Y. 14850, USA 
